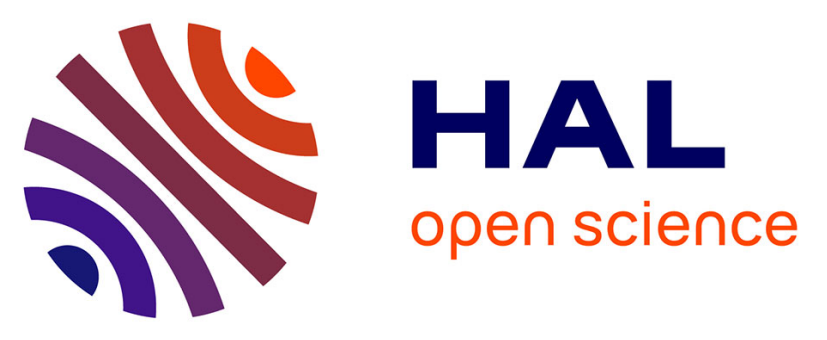

\title{
In-flight calibration of the Cassini-Radio and Plasma Wave Science (RPWS) antenna system for direction-finding and polarization measurements
}

Dieter F Vogl, Baptiste Cecconi, Wolfgang Macher, Philippe Zarka, Hans Peter Ladreiter, Pierre Fédou, Alain Lecacheux, Terry Averkamp, Georg Fischer, Helmut O Rucker, et al.

\section{To cite this version:}

Dieter F Vogl, Baptiste Cecconi, Wolfgang Macher, Philippe Zarka, Hans Peter Ladreiter, et al.. Inflight calibration of the Cassini-Radio and Plasma Wave Science (RPWS) antenna system for directionfinding and polarization measurements. Journal of Geophysical Research Space Physics, 2004, 109 (A9), pp.A09S17. 10.1029/2003JA010261 . hal-03274648

\section{HAL Id: hal-03274648 https://hal.science/hal-03274648}

Submitted on 30 Jun 2021

HAL is a multi-disciplinary open access archive for the deposit and dissemination of scientific research documents, whether they are published or not. The documents may come from teaching and research institutions in France or abroad, or from public or private research centers.
L'archive ouverte pluridisciplinaire HAL, est destinée au dépôt et à la diffusion de documents scientifiques de niveau recherche, publiés ou non, émanant des établissements d'enseignement et de recherche français ou étrangers, des laboratoires publics ou privés. 


\title{
In-flight calibration of the Cassini-Radio and Plasma Wave Science (RPWS) antenna system for direction-finding and polarization measurements
}

\author{
Dieter F. Vogl, ${ }^{1}$ Baptiste Cecconi, ${ }^{2}$ Wolfgang Macher, ${ }^{1}$ Philippe Zarka, ${ }^{2}$ \\ Hans Peter Ladreiter, ${ }^{1}$ Pierre Fédou, ${ }^{2}$ Alain Lecacheux, ${ }^{2}$ Terry Averkamp, ${ }^{3}$ Georg Fischer, ${ }^{1}$ \\ Helmut O. Rucker, ${ }^{1}$ Donald A. Gurnett, ${ }^{3}$ William S. Kurth, ${ }^{3}$ and George B. Hospodarsky ${ }^{3}$ \\ Received 30 September 2003; revised 10 February 2004; accepted 12 February 2004; published 9 July 2004.
}

[1] One major objective of the Cassini mission is the analysis of Saturnian radio emissions of magnetospheric (auroral) as well as atmospheric (lightning) origin. The Radio and Plasma Wave Science (RPWS) experiment is designed to measure the full polarization and the wave vector of the incoming radio waves, allowing us to retrieve information on source locations and emission modes. For that purpose, RPWS uses a two-channel receiver, connected to two electric monopoles (selected among three), which measures the voltages induced by the electric field of the incident waves and their various correlations. The accuracy of retrieved source locations depends directly on the precise knowledge of the orientation of the three effective monopole axes and lengths, which do not coincide with the physical ones owing to interaction with the spacecraft body. Antenna calibration aims at determining the so-called effective length vector of each antenna (combining orientation and length information). For that purpose, roll maneuvers of the Cassini spacecraft were performed before and after the Jupiter flyby, at distances such that Jovian radio sources can be identified with the planet's center but still provide a high signal-to-noise ratio. The resulting modulations of the measured signals allow us to derive the orientation and length of the effective antennas. The analysis is performed in two steps: first, the Stokes parameters (wave polarization) are determined using approximate antenna orientations derived from laboratory measurements on a scale model of the spacecraft. Second, measurements with high signal-to-noise ratio and pure circular polarization are selected and used for the determination of the effective length vectors of the RPWS antennas. Two methods have been developed for inverting the system of equations relating antenna parameters, wave parameters, and measurements (least squares fit and analytical inversion), both of which provide consistent results and present different advantages and limitations which are discussed. A final set of antenna parameters to be used for direction finding studies with the RPWS experiment is obtained. INDEX TERMS: 0609 Electromagnetics: Antennas; 6969 Radio Science: Remote sensing; 7534 Solar Physics, Astrophysics, and Astronomy: Radio emissions; 6994 Radio Science: Instruments and techniques; 6944 Radio Science: Nonlinear phenomena; KEYWORDS: antenna calibration, direction-finding, Cassini/RPWS, wave polarization, effective length vectors

Citation: Vogl, D. F., et al. (2004), In-flight calibration of the Cassini-Radio and Plasma Wave Science (RPWS) antenna system for direction-finding and polarization measurements, J. Geophys. Res., 109, A09S17, doi:10.1029/2003JA010261.

\section{Introduction}

[2] In 1997 the Cassini spacecraft was launched carrying 18 scientific instruments to investigate the Saturnian system

\footnotetext{
${ }^{1}$ Space Research Institute, Austrian Academy of Sciences, Graz, Austria.

${ }^{2}$ Laboratoire d'Etudes Spatiales et d'Instrumentation en Astrophysique, Observatoire de Paris, Meudon, France.

${ }^{3}$ Department of Physics and Astronomy, University of Iowa, Iowa City, Iowa, USA.

Copyright 2004 by the American Geophysical Union. 0148-0227/04/2003JA010261\$09.00
}

and its largest moon Titan. One major objective of the Cassini mission is the analysis of various types of Saturnian radio emissions, i.e., magnetospheric (auroral) as well as atmospheric (lightning) emissions, which will be recorded by the Radio and Plasma Wave Science (RPWS) experiment. This experiment uses a set of receivers connected to three nearly orthogonal electric antenna elements $u, v$, and $w$ (as illustrated in Figure 1) for electric field measurements, three orthogonal search coil magnetometers for magnetic field measurements, and a Langmuir probe for electron density and temperature measurements (a detailed description of the receivers as well as the instrumental setup is given by Gurnett et al. [2004]. The portion of the instru- 

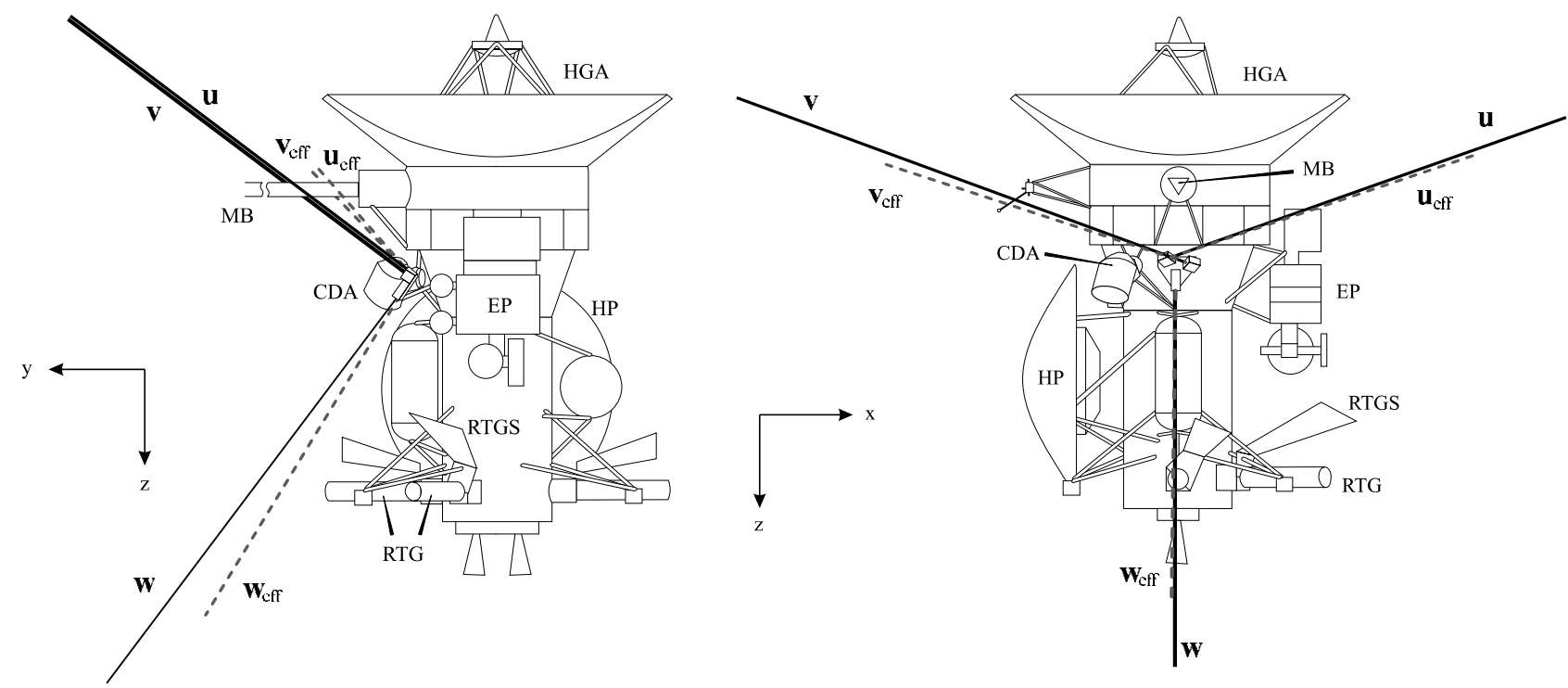

Figure 1. Sketch of the Cassini spacecraft adapted from Rucker et al. [1996]. Abbreviations are Magnetometer Boom (MB), Cosmic Dust Analyzer (CDA), Huygens Probe (HP), shades of the Radioisotope Thermoelectric Generators (RTG-S), Experimental Platform (EP), and High-Gain Antenna (HGA). The mechanical antenna elements are drawn as solid lines, and the corresponding effective antenna axes derived in this paper are shown as dashed lines.

ment of interest here is the High-Frequency Receiver (HFR), which consists of a pair of identical receivers analyzing the received signal in a number of frequency channels tunable from $3.5 \mathrm{kHz}$ up to $16 \mathrm{MHz}$. The two receivers are connected to two electric antennas selected among the three electric monopoles (for more details, see Gurnett et al. [2004]).

[3] In the standard "survey" analysis mode, the $u$ and $v$ monopoles are combined into an " $x$ " electric dipole, connected to one receiver, while the second receiver is connected to the $w$ monopole. The special mode of operation of interest here is the so-called direction-finding (DF) mode, in which the HFR receivers switch between the $(u, w)$ and the $(v, w)$ pairs of electric monopoles at each measured frequency. Simultaneous measurements of complex voltage autocorrelation and cross-correlations are performed for each pair of antennas. The real and imaginary part of the correlations can be expressed as a function of the wave polarization, the wave vector $(\mathbf{k})$ orientation, i.e., the source direction, and the antenna geometry.

[4] The accuracy of the wave DF technique will directly depend on the accuracy of the antenna parameters. While the geometrical length and orientation of the physical monopoles are very well known by construction [Gurnett et al., 2004], this is not the case for the electrically effective antennas, whose offsets with respect to the physical monopoles result from their interaction with the conducting spacecraft body. The resulting antenna axes and lengths are equivalent, at low frequencies where the antennas are short compared with the wavelength, to perfect electric dipoles with orientations and lengths somewhat different from the physical antennas. The most important quantity in dealing with short antennas is the effective length vector, representing the directional dependence of the reception properties of an antenna as well as the effective antenna length. It is thus necessary to determine with maximum accuracy the nine antenna parameters (two angles and a length for each of the three antennas) to ensure precise wave DF capability for the RPWS instrument.

[5] A first attempt at determining the effective length vectors of the RPWS antennas was done by Rucker et al. [1996] based on the concept of rheometry, an experimental determination of effective length vectors of antennas. This method consists in performing electrolytic tank measurements with a scale model of the antenna spacecraft system. A completely different approach is based on wire-grid modeling of the spacecraft body and antennas using computer codes (e.g., the Antenna Scatterers Analysis Program, ASAP, or the Numerical Electromagnetic Code, NEC) to solve the governing field integral equations for the current distribution on the spacecraft and the antennas [see Fischer et al., 2001], from which the effective axes are determined as the direction of minimum gain. Table 1 summarizes the corresponding results. Note that no effective lengths of the antennas are given because both techniques were applied assuming open feeds with no base capacitances connected. Nevertheless, these approaches yield realistic effective axes (directions). A realistic estimation of an effective antenna length can only be obtained taking into account capacitances: the measured voltages are determined by a capacitive divider consisting of the base and the antenna capacitance as described by Gurnett et al. [2004]. In-flight calibration thus overcomes these difficulties, since the whole reception system, consisting of receivers, antennas and interfering spacecraft body, is implicitly taken into account.

[6] In parallel, Ladreiter et al. [1995] developed an inversion method based on the singular value decomposition technique (SVD) to solve iteratively the set of nonlinear equations relating measurements to wave and antenna 
Table 1. Physical Directions and Effective Antenna Axes of the RPWS Antenna System Obtained by Rheometry Measurements and Wire-Grid Modeling With and Without the Huygens Probe $(\mathrm{HP})^{\mathrm{a}}$

\begin{tabular}{|c|c|c|c|}
\hline Quantity & $u$ Antenna & $v$ Antenna & $w$ Antenna \\
\hline \multicolumn{4}{|c|}{ Physical Direction } \\
\hline$h / h_{w}$ & 1.0 & 1.0 & 1.0 \\
\hline$\theta$ & $107.5^{\circ}$ & $107.5^{\circ}$ & $37.0^{\circ}$ \\
\hline$\phi$ & $24.8^{\circ}$ & $155.2^{\circ}$ & $90.0^{\circ}$ \\
\hline \multicolumn{4}{|c|}{ Rheometry (HP On) } \\
\hline$h / h_{w}$ & 0.91 & 0.91 & 1.0 \\
\hline$\theta$ & $107.9^{\circ}$ & $107.3^{\circ}$ & $31.4^{\circ}$ \\
\hline$\phi$ & $16.5^{\circ}$ & $162.7^{\circ}$ & $91.2^{\circ}$ \\
\hline \multicolumn{4}{|c|}{ Rheometry (HP Off) } \\
\hline$h / h_{w}$ & 0.91 & 0.91 & 1.0 \\
\hline$\theta$ & $107.6^{\circ}$ & $106.4^{\circ}$ & $30.8^{\circ}$ \\
\hline$\phi$ & $16.3^{\circ}$ & $163.5^{\circ}$ & $92.9^{\circ}$ \\
\hline \multicolumn{4}{|c|}{$A S A P(H P$ On) } \\
\hline$h / h_{w}$ & - & - & - \\
\hline$\theta$ & $106.4^{\circ}$ & $106.6^{\circ}$ & $29.6^{\circ}$ \\
\hline$\phi$ & $16.0^{\circ}$ & $164.2^{\circ}$ & $89.5^{\circ}$ \\
\hline \multicolumn{4}{|c|}{ ASAP (HP Off) } \\
\hline$h / h_{w}$ & - & - & - \\
\hline$\theta$ & $106.2^{\circ}$ & $105.7^{\circ}$ & $28.4^{\circ}$ \\
\hline$\phi$ & $15.3^{\circ}$ & $165.6^{\circ}$ & $91.7^{\circ}$ \\
\hline
\end{tabular}

${ }^{a}$ After Rucker et al. [1996] and Fischer et al. [2001]. Angles $\theta$ and $\phi$ are the colatitude and azimuth (in degrees) with respect to the coordinate system as shown in Figure 1. Note that for ASAP simulations, the effective length ratios are strongly dependent on the capacitative load at the antenna feeds.

parameters. These authors performed simulation studies of the effect of measurement accuracy and of the geometrical position of the source relative to the antennas on the antenna parameter determination.

[7] So far no measurements from the Cassini spacecraft have been included in all the mentioned approaches yet. As is discussed in the next section, we use radio wave observations of Jupiter during the Cassini Jupiter flyby to derive the effective length vectors of the RPWS antennas. Section 3 gives a description of the data processing. The mathematical description regarding the DF technique is then presented in section 4 . Two approaches regarding the antenna calibration mode are discussed in section 5. Finally, the last section is devoted to conclusions and outlook.

\section{Overview of the Cassini-RPWS Antenna Calibration}

[8] It was planned by the RPWS team to take advantage of the December 2000 flyby of Jupiter assuming Jupiter to be a point-like source of radio emissions with stable polarization characteristics. Jupiter is known to possess several intense radio sources of auroral origin: the broadband kilometer (bKOM), the hectometer (HOM), and the decameter (DAM) emissions. In addition, the interaction between Io and Jupiter induces specific decameter emissions (Io-DAM) [see, e.g., Zarka, 1998].

[9] As an illustrative example, Figure 2 shows the dynamic spectrum from the roll maneuver done by the Cassini spacecraft on 15 November 2000. Various emission types are detected: the decameter component ("DAM"), the hectometer component ("HOM"), the auroral broadband kilometer component ("bKOM"), and the narrowband emission ("nKOM"). The calibration sources and the distance of the observation must be chosen in a way that (1) the radio sources are located within $\approx 0.5^{\circ}$ of Jupiter's center so that the source direction may be taken with a good accuracy as the position of Jupiter's center and (2) radio emissions are observed with a signal-to-noise ratio $\geq 10 \mathrm{~dB}$. These two conditions led to define an optimum range of a Cassini-Jupiter distance of 300 to $600 R_{J}\left(R_{J}=\right.$ Jupiter radius $\left.=71,400 \mathrm{~km}\right)$ to perform the antenna calibration (AC).

[10] From the known average spectrum of Jupiter's radio components [see, e.g., Zarka, 1998], the signal-to-noise ratio (SNR) is expected to exceed $10 \mathrm{~dB}$ at distances up to $600 R_{J}$. Sources located at $r \leq 3 R_{J}$ from Jupiter's center are seen at a maximum angular distance of $\approx 0.6^{\circ}$ at $300 R_{J}$ and $\approx 0.3^{\circ}$ at $600 R_{J}$. Therefore $r \leq 3 R_{J}$ is a reasonable restriction for sources to get suitable angular accuracy for the antenna calibration. With an electron gyrofrequency of about 25 to $30 \mathrm{MHz}$ at the surface of the planet at high latitudes, decreasing with $R^{-3}$ in the distance (dipolar field), a maximum distance of $3 R_{J}$ corresponds to a minimum frequency of about $1 \mathrm{MHz}$. To be on the safe side, we will not use data with frequencies less than $600 \mathrm{kHz}$ in this work.

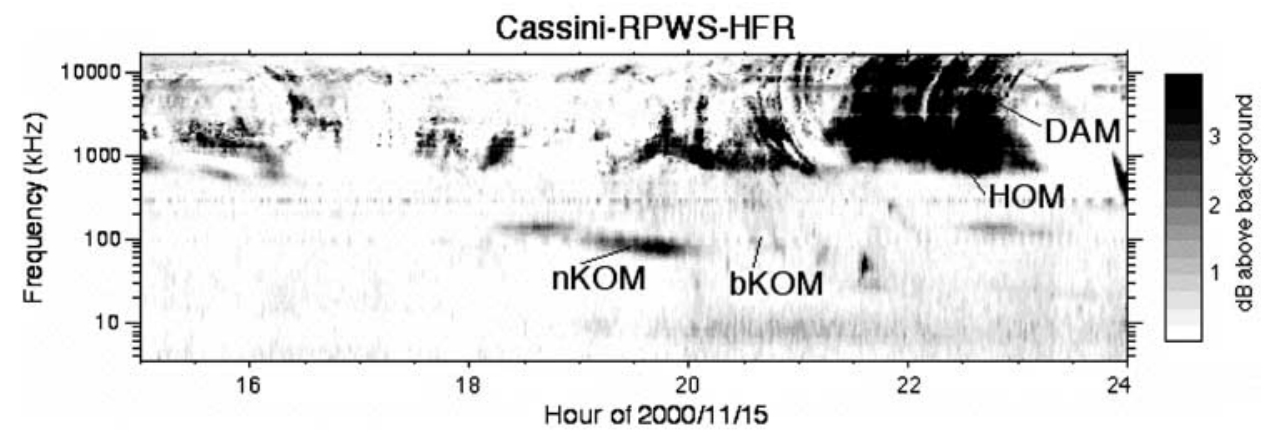

Figure 2. Dynamic spectrum as observed by Cassini on 15 November 2000 indicating various emission types from Jupiter: the decameter component ("DAM"), the hectometer component ("HOM"), the auroral broadband kilometer component ("bKOM"), and the narrowband emission ("nKOM"). 
Table 2. List of Roll Maneuvers Performed by Cassini for the Purpose of RPWS Antenna Calibration ${ }^{\mathrm{a}}$

\begin{tabular}{ccc}
\hline Date (Year DOY) & SCET (Hour: Begin-End) & Antennas \\
\hline & Inbound \\
2000320 & $15-25$ & $u, v$ \\
2000325 & $15-25$ & $u, v$ \\
2000330 & $15-24$ & $u, v$ \\
2000335 & $14-24$ & $u, v$ \\
2000340 & $14-24$ & $u, v$ \\
& & \\
2001022 & Outbound & $w$ \\
2001027 & $01-11$ & $w$ \\
2001036 & $00-11$ & $w$ \\
2001037 & $13-23$ & $w$ \\
\hline
\end{tabular}

${ }^{\mathrm{a}}$ Antennas passing near the source direction during the roll maneuvers are indicated in the third column.

[11] Within the mentioned distance range, a series of spacecraft rolls (duration $\approx 1$ hour/roll) were performed for the purpose of RPWS antenna calibration. During these roll maneuvers, one or several of the three antennas are expected to point close to the source direction, implying strong variations of the antenna's response to incoming radio waves (an electric dipole has power response proportional to $\sin ^{2}(\theta)$, with $\theta$ being the angle between the $\mathbf{k}$ vector of the incoming wave and the dipole axis, hence a zero response along its axis). This configuration has been shown to be an optimum for the position angles $\left(\theta_{i}, \phi_{i}\right)$ (for $\left.i=u, v, w\right)$ of the corresponding antenna [see Ladreiter et al., 1995] from the view of maximum angular sensitivity of the measurements, but it is also the position of minimum voltage sensitivity and so of largest bias due to background noise and not complete dipole-like behavior of the antennas. Therefore appropriate data selection is a delicate issue (see below). A total of nine roll maneuvers of the spacecraft were performed along the inbound and outbound trajectories, as listed in Table 2. During these roll maneuvers, the HFR performed continuously 30-s sweeps of its whole frequency range, from $3.5 \mathrm{kHz}$ up to $16.125 \mathrm{MHz}$, in the antenna-switching (DF) mode.

\section{Data Processing and Preselection}

[12] A full set of DF data at any HFR frequency, consisting of $(u, w)$ and $(v, w)$ consecutive measurements, corresponds to a set of autocorrelations $\left\langle V_{i} V_{i}^{*}\right\rangle$ with $i=u, v$, $w$ (noted as $A_{u u}, A_{v w}$ and $A_{w w}$ ) and two pairs of real and imaginary parts of cross-correlation signals accordingly noted as $C_{u w}, C_{v w}, I_{u w}$, and $I_{v w}$. These values, corresponding to seven equations (one per measurement) and depending on six wave parameters $(S, Q, U, V, \theta$, and $\phi)$ and nine antenna parameters $\left(h_{i}, \theta_{i}, \phi_{i}\right.$, with $\left.i=u, v, w\right)$, are considered to be measured simultaneously, while they consist in fact of two subsets of four measurements each $\left(A_{w w}\right.$ is obtained twice, thus denoted as $A_{w w}$ and $\tilde{A}_{w w}$ ) separated by 10 to $160 \mathrm{~ms}$ depending on the HFR status. The tilde sign refers to the second subset of measurements.

[13] Assuming that the polarization of the observed radio emissions does not change between the two subsets of measurements, the mean value of the two $A_{w w}$ measurements could be used. However, the observed signal might be the sum of different fluctuating sources [see OrtegaMolina and Lecacheux, 1991], leading to a change of its apparent state of polarization between measurement subsets. Therefore we use both subsets of measurements separately. This is a much more favorable situation than the DF measurements performed on a rotating spacecraft because we need not the assumption that the observed radio source has a stable emission during a complete roll period (some seconds for fast-spinning spacecraft; 1 hour in the case of the Cassini spacecraft) which is generally not true [see, e.g., Ladreiter et al., 1994]. Information on the Stokes parameters $S$ (characterizing the wave intensity), $Q$ and $U$ (characterizing linear polarization), and $V$ (characterizing circular polarization) is essential to deduce source brightness temperature, emitted power, and mode of emission.

[14] In Figure 3 we show as an illustrative example the induced voltages on the RPWS antenna system during the inbound roll maneuver done on 15 November (DOY 320) 2000 , as a function of spacecraft event time (SCET). From top to bottom we plot the frequencies (in $\mathrm{kHz}$ ) of the observed electromagnetic wave intensities, the autocorrelations, $A_{u u}, A_{v v}, A_{w w}$, and $\tilde{A}_{w w}$ (in $\mathrm{V}^{2} / \mathrm{Hz}$ ) and the normalized cross-correlation signals $C_{u w}, C_{v w} I_{u w}$ and $I_{v w}$, respectively. The normalization of the cross-correlations is done for convenience, e.g., quantity $C_{u w}$ is divided by $\sqrt{A_{u u} A_{w w}}$. Note that the measurements correspond to a signal-to-noise ratio greater than $20 \mathrm{~dB}$ within the full frequency range of $475 \mathrm{kHz}$ up to $4075 \mathrm{kHz}$. The autocorrelations are plotted in units of $10^{-15} \mathrm{~V}^{2} / \mathrm{Hz}$ and a background noise, whose determination is discussed by Zarka et al. [this issue], is subtracted from the data (see also Appendix A).

[15] In the Cassini coordinate system the model-predicted values are given as (after Ladreiter et al. [1995])

$$
\begin{aligned}
\Re\left(\left\langle V_{i} V_{j}^{*}\right\rangle\right)= & \frac{S h_{i} h_{j}}{2}\left[(1+Q) \Omega_{i} \Omega_{j}-U \sin \theta_{i} \sin \left(\phi-\phi_{i}\right) \Omega_{j}\right. \\
& -U \sin \theta_{j} \sin \left(\phi-\phi_{j}\right) \Omega_{i}+(1-Q) \\
& \left.\cdot \sin \theta_{i} \sin \theta_{j} \sin \left(\phi-\phi_{i}\right) \sin \left(\phi-\phi_{j}\right)\right]
\end{aligned}
$$

and

$$
\begin{aligned}
\Im\left(\left\langle V_{i} V_{j}^{*}\right\rangle\right)= & \frac{S h_{i} h_{j}}{2} V\left[\sin \theta_{i} \sin \left(\phi-\phi_{i}\right) \Omega_{j}\right. \\
& \left.-\sin \theta_{j} \sin \left(\phi-\phi_{j}\right) \Omega_{i}\right] .
\end{aligned}
$$

Here, $\Omega_{i}=\cos \theta_{i} \sin \theta-\sin \theta_{i} \cos \theta \cos \left(\phi-\phi_{i}\right)$, the quantity $V_{i}$ represents the analytic signals of the voltages at each receiver input [see Born and Wolf, 1993], 〈... denotes the time-averaging operation, and the asterisk refers to the complex conjugate. Parameters $\theta$ and $\phi$ are the colatitude and azimuth of the source direction in the spacecraft reference frame, quantities $\theta_{i}$ and $\phi_{i}$ are the colatitude and azimuth of the corresponding electric antennas, and $h_{i}$ are their effective lengths, respectively. The angular position of Jupiter is obtained by using the orbit position of Cassini in the Jupiter-Solar-Ecliptic (JSE) coordinate system ( $X$ points from Jupiter to the Sun, $Z$ is normal to Jupiter's orbital plane, and $Y$ completes the right-handed triad). 


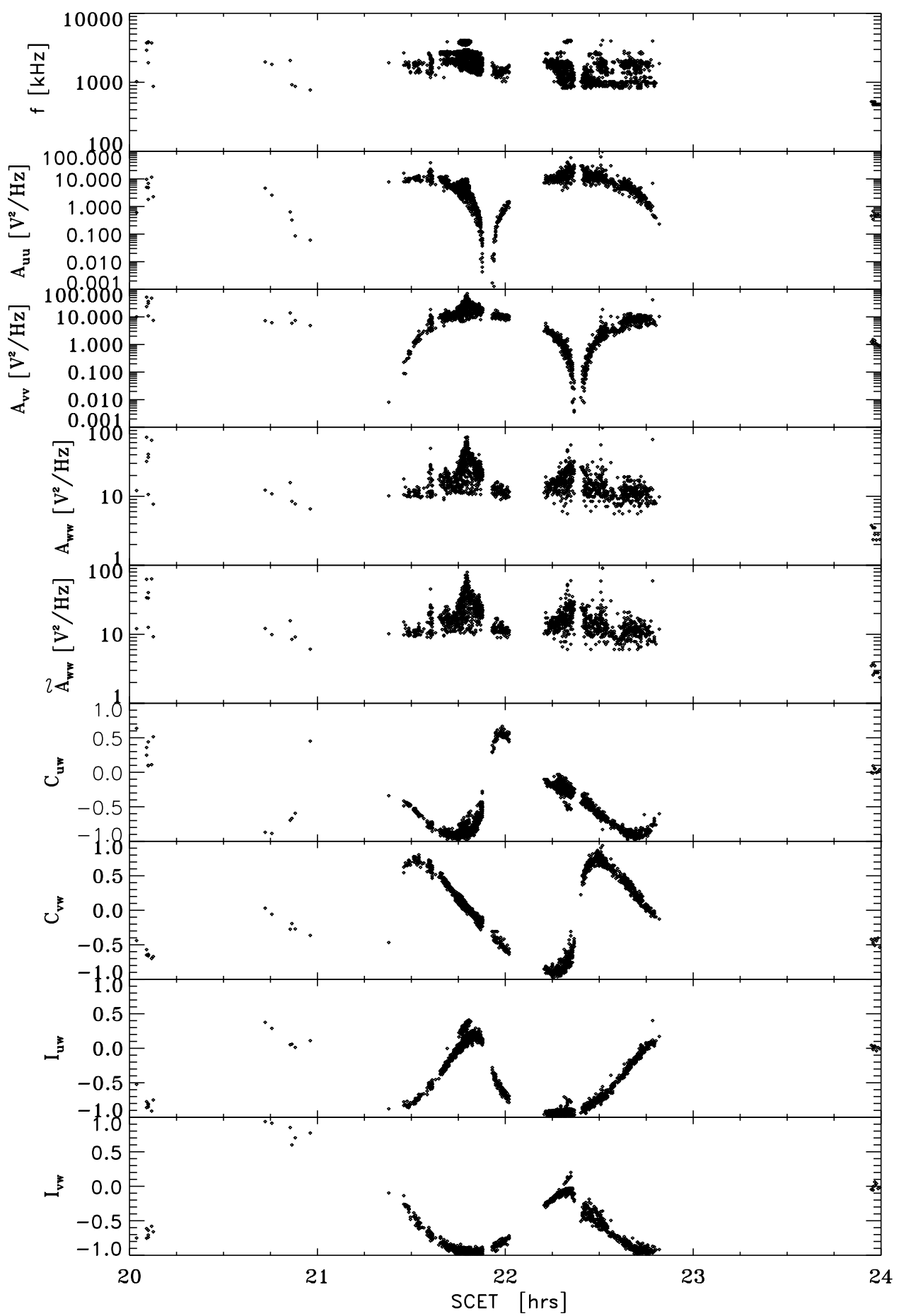

Figure 3. Autocorrelation and cross-correlations as observed by the Cassini spacecraft on 15 November (DOY 320) 2000 (first roll maneuver according to Table 2). From top to bottom we show the frequencies within the range $475 \leq f \leq 4075 \mathrm{kHz}$, and the autocorrelation and normalized cross-correlations as a function of spacecraft event time (SCET). The autocorrelations are plotted in units of $10^{-15} \mathrm{~V}^{2} / \mathrm{Hz}$. Note that the measurements correspond to a signal-to-noise ratio greater than $20 \mathrm{~dB}$.

[16] For both the DF and the AC analysis, we search for solutions, where the weighted least squares sum of the difference between the wave observations, $y_{i}^{\text {obs }}(i$ autocorrelation and cross-correlation measured by the RPWS antenna system, as shown in Figure 3), and the model- predicted values, $y_{i}^{\bmod }$ becomes a minimum [see Ladreiter et al., 1995],

$$
\chi^{2}=\sum_{i=1}^{N} \mathcal{W}_{i}\left[y_{i}^{\text {obs }}-y_{i}^{\text {mod }}(\mathbf{X})\right]^{2}=\text { Min } .
$$



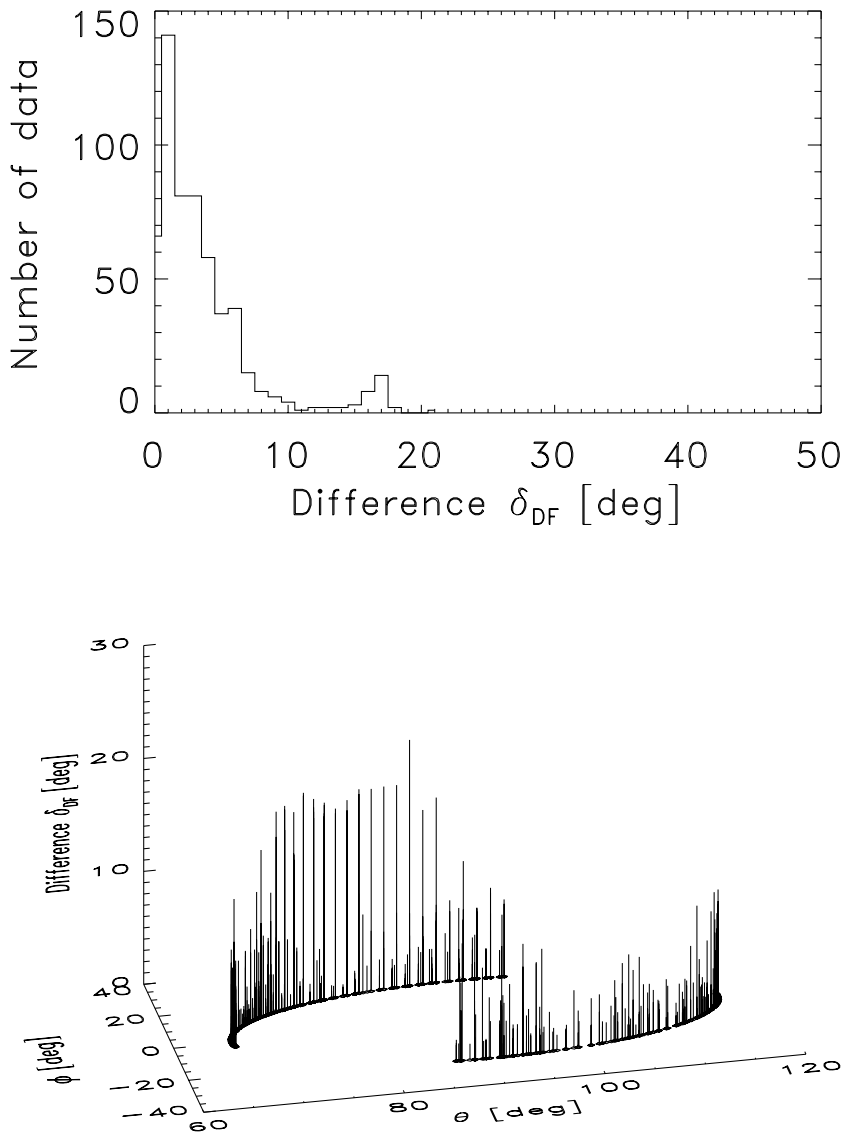

Figure 4. Difference (solid lines) between the DF retrieved values and Jupiter's position. The top panel shows the error occurrences as a function of the error magnitude. The bottom panel gives these errors as a function of the source position (ephemeris data).

Here, the quantity $\mathbf{X}$ contains the wave parameters $S, Q, U$, $V, \theta$, and $\phi$ (DF mode) or the antenna parameters $h_{u} / h_{w}, \theta_{u}$, $\phi_{u}, h_{v} / h_{w}, \theta_{v}, \phi_{v}, \theta_{w}$, and $\phi_{w}$, respectively (AC mode). Note that only the ratios of the lengths of the $u$ and $v$ antennas with respect to the $w$ antenna can be obtained because the intensity $(S)$ of the incident waves is not known a priori and appears as a multiplying factor. Quantity $\mathcal{W}$ refers to the weights reflecting the uncertainty of each measurement, $\mathcal{W}=\sigma^{-2}$, where $\sigma$ is the standard deviation of the corresponding measurements, which includes the intrinsic receiver noise level of a maximum of $\sim 10^{-16} \mathrm{~V}^{2} / \mathrm{Hz}$ [see Gurnett et al., 2004; Zarka et al., this issue].

[17] Before proceeding in the analysis we preselect only measurements where the angular distances, $\delta$, from the position of Jupiter (ephemeris data) to each RPWS antenna is at least $15^{\circ}$,

$$
\delta_{i}=\arccos \left[\sin \theta \sin \theta_{\text {rheo }, i} \cos \left(\phi_{\text {rheo }, i}-\phi\right)+\cos \theta \cos \theta_{\text {rheo }, i}\right] .
$$

Here, quantities $\theta_{\text {rheo, } i}$ and $\phi_{\text {rheo }, i}$ represent the angles of the respective effective axes from the rheometry experiment (see Table 1) and $\theta$ and $\phi$ are the Jupiter ephemeris. As discussed above, this criterion is applied to exclude data observed when one of the antennas is quite insensitive and so great errors due to noise and nonapplicability of the quasi-static length vector occur. The latter effect plays a role for all directions, anyway, if the frequencies are to high, i.e., if the wave length is not much greater that antenna dimensions. Therefore another preselection of the observations is done with respect to the frequency range, namely a restriction to the frequency interval $0.6<f<1.35 \mathrm{MHz}$. At higher frequencies the effective length vectors become complex, with both direction and frequency dependencies [Ortega-Molina and Daigne, 1984].

[18] So the whole procedure of in-flight calibration can be shortly summarized as follows: (1) Data preselection: $0.6<f<1.35 \mathrm{MHz}$, angle between Jupiter and each antenna axes $>15^{\circ}$, SNR $>20 \mathrm{~dB}$. (2) Computation of wave parameters (Stokes parameters and direction of the incident waves) by keeping the antenna parameters fixed, i.e., the rheometry values are used as input parameters, further assuming the source to be located at the center of Jupiter. (3) Data selection with regard to the state of polarization and direction of the incident waves. (4) The actual calibration deals with the computation of the effective length vectors by setting the wave parameters to fixed known values (superposition of circularly polarized waves) using a least squares fit and an analytical inversion method.

\section{Direction-Finding Analysis}

[19] In the DF analysis we search for the parameters $Q, U$, $V, \theta$, and $\phi$ by keeping the values of the effective length vectors constant, namely the results from the rheometry experiment are used as input parameters (see Table 1). We also assume that the state of polarization does not change much between the two subsets of measurements; therefore we define the quantity $\bar{A}_{w w}$ as the mean value of the autocorrelations $A_{w w}$ and $\tilde{A}_{w w}$ of both subsets of measurements. Starting values for the wave parameters are $Q=0$, $U=0, V=0$, and the ephemeris data for the directions of the incident waves.

[20] As an illustrative example, Figure 4 shows the difference (in degrees) between the DF retrieved values and the ephemeris data, $\theta$ and $\phi$ for all inbound observations (see Table 2). The ephemeris data are plotted as black dots in the $\phi-\theta$ plane (bottom). The length of each solid line gives the corresponding angular difference $\delta_{D F}$ (in degrees) between the computations and Jupiter's position. The histogram (top of the figure) shows the distribution of the angular errors. As it is seen from the figure, some DF results considerably deviate from the ephemeris. The small peak above 15 degrees corresponds to sporadic emissions from other sources, e.g., solar type III emissions. Therefore we only consider those voltage measurements where this difference is less than 10 degrees, which contains $93 \%$ of the data. Within this interval, $80 \%$ of the data show an error less than 5 degrees. This error distribution will be typical for future direction finding analysis based on the antenna parameters determined by in-flight calibration.

[21] Another selection is done with respect to the state of polarization. We use the so-called degree of linear polarization, $P_{l i n}=\sqrt{Q_{D F}^{2}+U_{D F}^{2}}$, and select the RPWS measurements for $P_{l i n}$ being less than 0.2. This is justified 

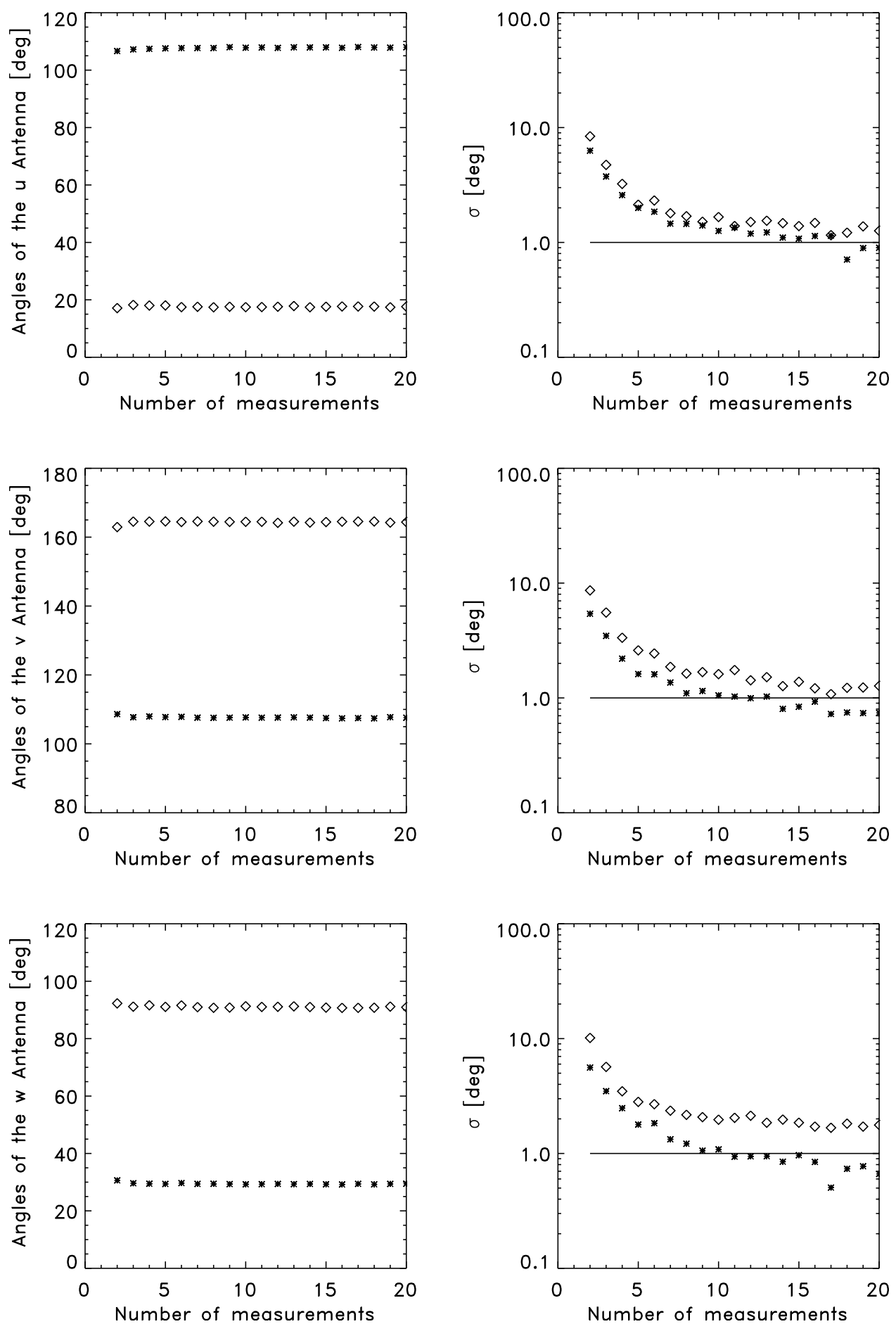

Figure 5. The left panels show averaged values of the colatitude (star symbol) and azimuth (diamond symbol) for the $u$ antenna (top), the $v$ antenna (middle), and the $w$ antenna (bottom) versus the number of data sets to be used. The right panels give the corresponding standard deviations.

by former observations of the Hectometer emission of Jupiter (for more detail see Ortega-Molina and Lecacheux [1991]). We note that all these restrictions (frequency range, signal-to-noise ratio, distance between Jupiter's position and the DF retrieved values of colatitude and azimuth of wave incidence, degree of linear polarization) reduce all observations (according to Table 2) down to
1189 data sets, which are then finally used for the estimation of the effective length vectors.

\section{Antenna Calibration}

[22] Using the selected voltage measurements, we next focus on the estimation of the effective length vectors of the 

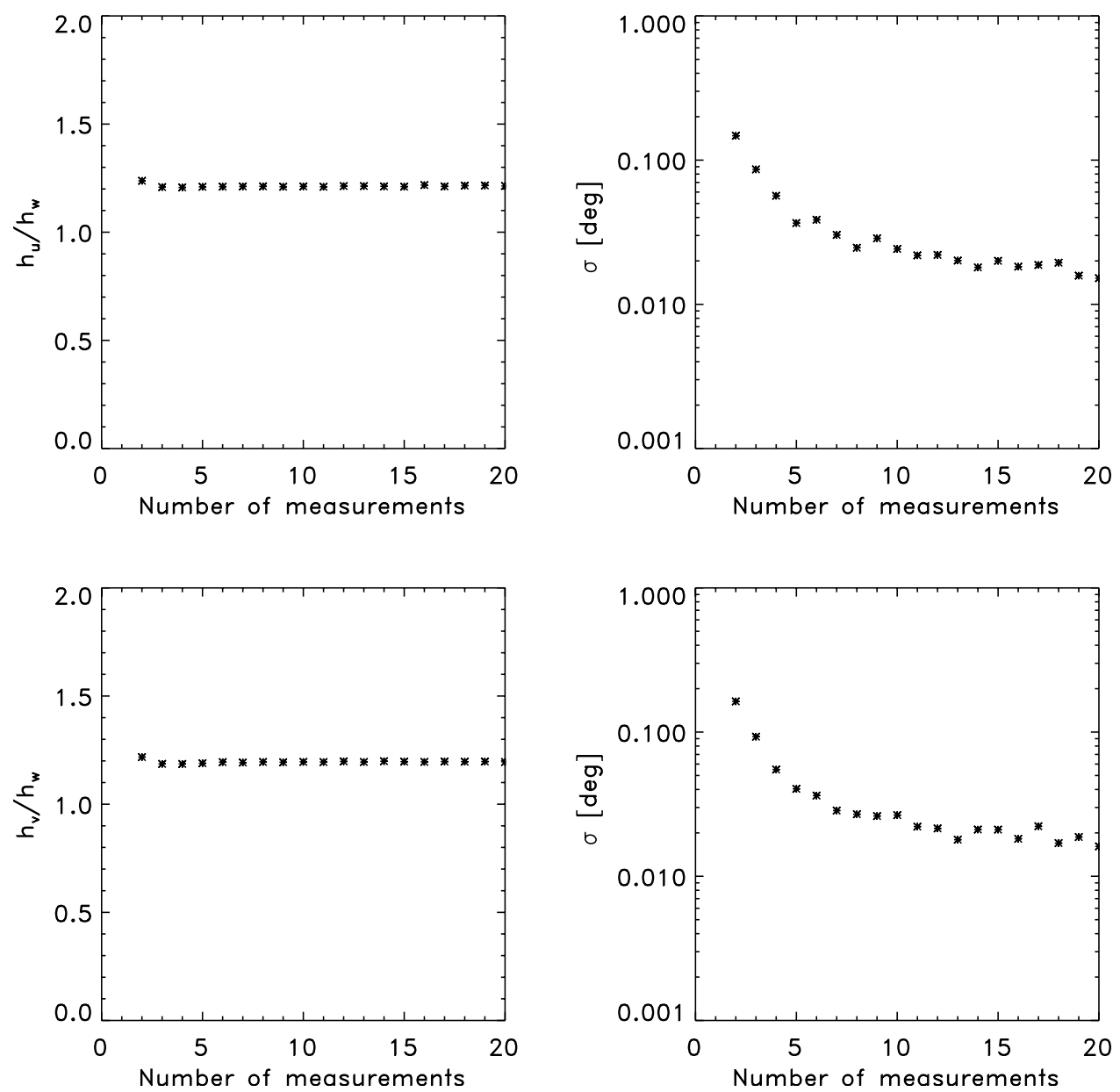

Figure 6. Length ratios of the RPWS antennas versus the number of data sets to be used.

RPWS antennas by introducing two different methods, a least squares fit and an analytical inversion method.

\subsection{Least Squares Fit Method}

[23] Similar to the DF analysis, we search for the weighted least squares difference between the observations and modeled quantities (equations (1) and (2)). The set of equations in the DF mode is overdetermined (seven equations are given for six unknown wave parameters), but this is not the case in the AC mode. For a set of autocorrelation and cross-correlations at a measured frequency, eight antenna parameters have to be derived using only seven equations. Our least squares model thus works as follows: We consider a possible changing of the state of polarization during the switching time of the two receivers and use both subsets of measurements. Moreover, we do not take into account the cross-correlations $I_{u w}$ and $I_{v w}$ according to equation (2), since it only introduces the parameter $V$ without any further information on the unknown antenna parameters. Additionally, the equations are normalized so that no information on the parameter $S$ is needed (dropping from the set of equations). The relevant mathematical formalism is given in detail in Appendix D.

[24] As a matter of fact, the problem is reduced to four equations for given eight unknown antenna parameters. Hence to avoid an underdetermined configuration we use
$M \geq 2$ sets of autocorrelation and cross-correlations simultaneously. These voltage measurements are furthermore randomly chosen from the measurement sets available after selection. Thus for $M=2$ at least (1) two inbound, or (2) two outbound, or (3) one inbound and one outbound measurement set are used in one step of computation. For a total of $N$ measurement sets we therefore get $N / M$ independent solutions for the antenna parameters (for simplicity we here assume that $N$ is a multiple of $M$ ), which defines one cycle of computations. For each $M$ (number of data to be used simultaneously) from 2 to 20 , similar computations are performed giving a total of 18 cycles of computations, the results of which are displayed in Figure 5.

[25] From top to bottom the left panels show the averaged values of the azimuth, $\phi$ (diamond symbol), and the colatitude, $\theta$ (star symbol) of the $u$ (top), $v$ (middle), and $w$ (bottom) antenna versus the number of data sets to be used. The right panels show the corresponding standard deviations of these angles versus the number of data sets to be used. For example if one takes 10 data sets simultaneously $(M=10)$, the corresponding single diamond and star symbol in each left panel represent the averaging of 118 estimations of the colatitude and azimuth of each antenna. The averaged values of the directions (and corresponding standard deviations) are then in this case $\theta_{u}=107.8^{\circ}\left(\sigma_{\theta_{u}}=1.3^{\circ}\right), \theta_{v}=107.7^{\circ}\left(\sigma_{\theta_{v}}=1.1^{\circ}\right), \theta_{w}=29.3^{\circ}$ 
Table 3. Colatitude, Azimuth, and Length of the RPWS Antennas as Derived From Induced Voltage Measurements During the Jupiter Flyby of Cassini Using the $\chi^{2}$ Method

\begin{tabular}{lcccccc}
\hline & $u$ Antenna & $( \pm \sigma)$ & $v$ Antenna & $( \pm \sigma)$ & $w$ Antenna & $( \pm \sigma)$ \\
\hline$h / h_{w}$ & 1.21 & $( \pm 0.02)$ & 1.19 & $( \pm 0.02)$ & 1.0 & $( \pm 0.0)$ \\
$\theta$ & $107.9^{\circ}$ & $\left( \pm 1.2^{\circ}\right)$ & $107.6^{\circ}$ & $\left( \pm 1.0^{\circ}\right)$ & $29.3^{\circ}$ & $\left( \pm 1.0^{\circ}\right)$ \\
$\phi$ & $17.6^{\circ}$ & $\left( \pm 1.5^{\circ}\right)$ & $164.4^{\circ}$ & $\left( \pm 1.5^{\circ}\right)$ & $90.9^{\circ}$ & $\left( \pm 2.0^{\circ}\right)$ \\
\hline
\end{tabular}

$\left(\sigma_{\theta_{w}}=1.1^{\circ}\right), \phi_{u}=17.5^{\circ}\left(\sigma_{\phi_{u}}=1.7^{\circ}\right), \phi_{v}=164.5^{\circ}\left(\sigma_{\phi_{v}}=1.6^{\circ}\right)$, and $\phi_{w}=91.3^{\circ}\left(\sigma_{\phi_{w}}=2.0^{\circ}\right)$, respectively. Note that the accuracy is improved with $M$.

[26] Analogously, Figure 6 gives the averaged values (left panels) and standard deviations (right panels) of the lengths of the $u$ antenna (top) and $v$ antenna (bottom) with respect to $h_{w}$ versus the number of data sets to be used (note that $h_{w}=$ $1.0)$. The computations clearly show that the $w$ antenna is shorter than the $u$ and $v$ antennas, quite different to the rheometry experiment. Again, using 10 data sets simultaneously, the lengths are $h_{u}=1.21\left(\sigma_{h_{u}}=0.03\right)$ and $h_{v}=1.19$ $\left(\sigma_{h v}=0.03\right)$. Similar results are deduced from observed resonance frequencies [cf. Zarka et al., this issue]. Initial guesses for iteration process to obtain the antenna axes are the physical axes, but we have checked that the same results are obtained by using the results from the rheometry experiment as initial guesses.

[27] We further found that a low number of data sets used yields a higher standard deviation and thus a bigger scatter of the derived angles and lengths. For an increase of the number of sets of used measurements, the standard deviation goes down to values between one and two degrees and stays fairly constant between the range of ten up to twenty used measurements. Note that the variance is proportional to $1 / N$. This proportionality is approximately given for a low number but not for a higher number of data sets to be used (see right panels in Figures 5 and 6). The asymptotic values of the standard deviations (between one and two degrees) is then due to systematic errors like the assumption that the radio source is emitted from the center of Jupiter (see section 3 ) or the oscillation of each antenna.

[28] Therefore we provide a best estimation for the effective length vectors by taking the average over all derived angles in the range of eight up to eighteen used measurements. The results are summarized in Table 3 .

\subsection{Analytical Inversion Method}

[29] Parallel to the least squares fit method, a fully analytical inversion (AI) method has been developed for both the direction-finding and the antenna calibration analysis. As seen from equations (1) and (2), the expressions of the modeled autocorrelation and cross-correlations are complicated in the spacecraft reference frame. For the sake of simplicity and without loss of generality, we change from the spacecraft reference frame to the so-called wave frame, in which the $\mathrm{z}$ axis is the normalized wave vector, $\mathbf{z}=\hat{\mathbf{k}}$. Then we choose the $\mathbf{y}$ axis in the plane containing the $\hat{\mathbf{k}}$ vector and Jupiter's rotation axis (with orientation along the SouthNorth direction. The $\mathbf{x}$ axis completes the right-hand triad. The geometry of the wave frame relative to the spacecraft frame is illustrated in Figure E1 (see Appendix E).

[30] The relevant equations are discussed in Appendix E. Antenna calibration is performed with two-antenna data sets, assuming $U=0$ and $Q=0$ (no linear polarization as in the previous method). A critical step conditioning the accuracy of the results is an appropriate data selection (according to signal-to-noise ratio, polarization, and especially source position relative to the antennas), which depends on the specific antenna parameter to be determined. One antenna is calibrated at a time, with appropriate data selection and assuming that we know the other antenna in the pair.

[31] The main benefit of the analytical inversion, besides the fact that it allows fast computation of results over large data sets, is that for each set of measurement there is one single solution. Errors on derived parameters can be related to errors on the measurements (analytically or through simulation).

[32] The analysis of these errors performed by B. Cecconi et al. (Direction-finding and antenna calibration through analytical inversion of radio measurements performed using a system of 2 or 3 electric dipole wire antennas, submitted to Radio Science, 2004, hereinafter referred to as Cecconi et al., submitted manuscript, 2004) reveals that some specific geometrical configurations (source $\leftrightarrow$ RPWS antennas) are very unfavorable for antenna calibration and thus lead to very large errors in the results. These configurations must be excluded in the process of data selection as discussed below. For example, with the selection criteria used below, $\mathrm{a} \pm 2^{\circ}$ error on the $u$ or $v$ antenna direction results in an error on the $w$ colatitude and azimuth of $3.5^{\circ}$ and $7^{\circ}$, respectively, and $2 \%$ on antenna length ratio. The final error given on the antenna parameters is directly linked to the width of the resulting cloud of points (see Figure 7).

[33] The selection criteria applied to the data incorporate several geometrical conditions in addition to those described in section 4 . We define $\alpha_{i}(i=u, v, w)$ as the angular distance between the $i$ th antenna and the source direction. We further define $\beta_{u}$ as the angular distances between the plane containing the $u$ and the $w$ antennas and the source direction. Similarly, $\beta_{v}$ is the angular distances between the plane containing the $v$ and the $w$ antennas and the source direction. With these definitions, geometrical selection criteria consist

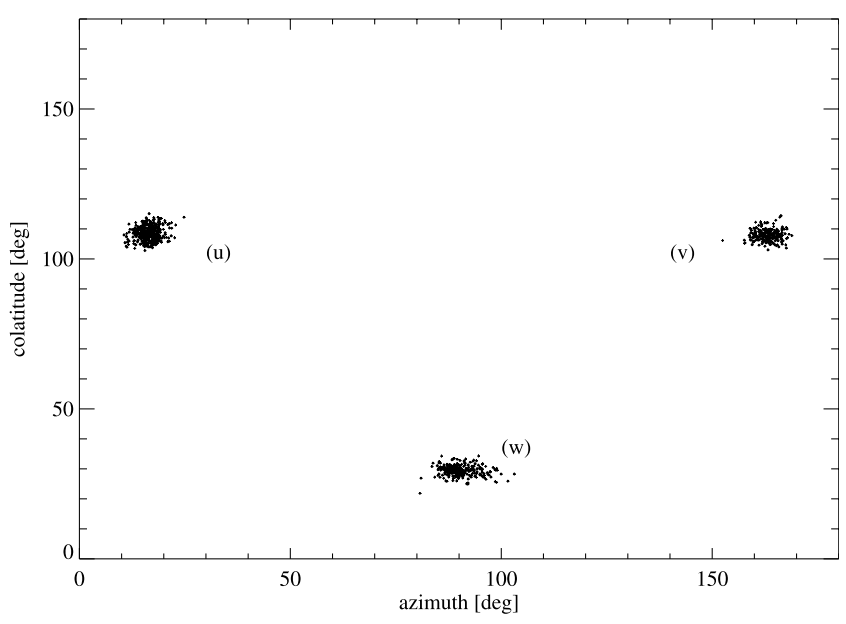

Figure 7. Results of the antenna calibration with the analytical inversion model. Each cloud of points has been derived analytically from the selected data sets described in the text. 
Table 4. Colatitude and Azimuth of the RPWS Antennas Derived by Analytical Inversion ${ }^{\text {a }}$

\begin{tabular}{lcccccc}
\hline & $u$ Antenna & $( \pm \sigma)$ & $v$ Antenna & $( \pm \sigma)$ & $w$ Antenna & $( \pm \sigma)$ \\
\hline$h / h_{w}$ & 1.20 & $( \pm 0.03)$ & 1.18 & $( \pm 0.05)$ & 1.0 & $( \pm 0.0)$ \\
$\theta$ & $108.5^{\circ}$ & $\left( \pm 2.3^{\circ}\right)$ & $108.0^{\circ}$ & $\left( \pm 1.9^{\circ}\right)$ & $29.4^{\circ}$ & $\left( \pm 1.6^{\circ}\right)$ \\
$\phi$ & $16.5^{\circ}$ & $\left( \pm 1.8^{\circ}\right)$ & $163.1^{\circ}$ & $\left( \pm 2.4^{\circ}\right)$ & $90.3^{\circ}$ & $\left( \pm 3.3^{\circ}\right)$ \\
\hline
\end{tabular}

${ }^{\mathrm{a}}$ Two values are given for the $w$ antenna. Since we do the calibration on two-antenna data sets, we can use either the $(u, w)$ or the $(v, w)$ pair of antennas.

in excluding (1) directions close to the antennas $\left(\alpha_{i}<15^{\circ}\right)$ resulting in low SNR and (2) directions perpendicular to the antenna to be calibrated $\left(\alpha_{i}>50^{\circ}\right)$, for which data poorly constrain the antenna angles.

[34] In addition, selections described in section 4 are made using the following thresholds: $\operatorname{SNR}\left(A_{w w}\right)>20 \mathrm{~dB}$ for $u$ or $v$ antenna calibration, and $\operatorname{SNR}\left(A_{i i}\right)>20 \mathrm{~dB}(i=u$, $v$ ) for $w$ antenna calibration, $P_{\text {lin }}<0.2$, and angular distance from the source direction to the position of Jupiter $<10^{\circ}$.

[35] We thus end with 479 four-measurement data sets for the $u$ antenna calibration, 243 data sets for the $v$ antenna calibration, and 288 for the $w$ antenna calibration. These data sets are extracted from all roll maneuvers according to Table 2. However, in the case of the $w$ antenna calibration, slightly different results are obtained for $\theta_{w}$ and $\phi_{w}$ with the $(u, w)$ pair of antennas (145 data sets leading to $\theta_{w}=29.0^{\circ} \pm$ $1.5^{\circ}$ and $\left.\phi_{w}=89.9^{\circ} \pm 2.6^{\circ}\right)$ and the $(v, w)$ pair of antennas (143 data sets leading to $\theta_{w}=29.7^{\circ} \pm 1.6^{\circ}$ and $\phi_{w}=90.7^{\circ} \pm$ $\left.4.0^{\circ}\right)$. The origin of these differences is not well understood. It could be due to residual systematic errors resulting from correlations between the antenna parameters in the fourequation sets not perfectly corrected by data selection.

[36] The results are summarized in Table 4 where averages are given for the $w$ antenna. Results for antenna colatitude and azimuth are plotted in Figure 7. Error bars in Table 4 correspond to the dispersions of the cloud of points.

\section{Discussion and Conclusion}

[37] We analyze voltage measurements induced by the RPWS antennas on board the Cassini spacecraft during several roll maneuvers at the Jupiter flyby to determine the effective length vectors of the three electric antennas. For that purpose two methods have been developed by the RPWS team, a least squares fit method and an analytical inversion technique. Several restrictions to the induced voltage measurements are made and applied to the models.

[38] First we restrict the autocorrelation and cross-correlations to a frequency range of $600<f<1350 \mathrm{kHz}$. This frequency range covers parts of the hectometer radio emissions from Jupiter. This frequency range is chosen since (1) at higher frequencies the effective length vectors become complex and at lower frequencies the source distance from Jupiter can be too large to have sufficient angular accuracy, (2) it is related to a superposition of circularly polarized electromagnetic waves, and (3) does not include any resonance between the antennas and the spacecraft body (quasi-static range). The analysis using the least squares method is performed in two steps: First, we derive the wave information, i.e., the Stokes parameters and the wave direction are obtained by keeping the antenna parameters constant (values for the antenna parameters are taken from the rheometry experiment). Second, considering the degree of linear polarization less than 0.2 (as derived from the DF analysis) the effective length vectors of the three antennas are determined by setting $Q=0$ and $U=0$ (for both methods).

[39] The least squares fit method includes the intrinsic receiver noise of a maximum of $10^{-16} \mathrm{~V}^{2} / \mathrm{Hz}$ for both, the direction-finding and the antenna calibration analysis. Several further restrictions to the set of observations are made: (1) the angular distance from the position of Jupiter (ephemeris data) to each RPWS antenna is at least $15^{\circ}$, (2) the distance between Jupiter's position and the derived values from the DF analysis is less than $10^{\circ}$, and (3) the angular distances between the plane containing the $u$ and the $w$ antennas and the source direction, $\beta_{i}$, is in the AI model additionally considered.

[40] In the second part of the analysis the effective length vectors of the three RPWS monopoles are derived assuming $Q=0$ and $U=0$ as input values. Moreover, we chose as an initial guess for the antenna parameters (lengths, colatitude, and azimuth) the physical directions, as reported in Table 1. Additionally, we take into account that the state of polarization might change between the switching time of the two receivers; therefore we use both subsets in the antenna calibration. The main benefits of the specific least squares method, i.e., fitting of normalized autocorrelation and crosscorrelations, are that (1) the total flux and the error from the automatic gain control of the receiver do not influence these computations and (2) up to 20 measurements are used simultaneously and randomly (various combinations of inbound and outbound measurements), giving well conditions for stable results. Furthermore, no assumptions on the antenna parameters are initially made in the actual calibration, so the results are independent of former applied methods.

[41] The derived values of the colatitude and azimuth, presented in Table 3, are compared with previous results. We found that the directions of the effective length vectors are slightly different from rheometric measurements and wire-grid modeling, but we emphasize that the results are consistent within the inherent standard deviations. The effective axis of the $w$ antenna is less accurately determined than those of the $u$ and $v$ antennas because the $w$ antenna is closer to the spacecraft body and therefore more influenced by the induced surface currents. Nevertheless, the direction of the $w$ antenna found by in-flight calibration is very close to the rheometric results. As no comparable effective length ratios from rheometry and wire-grid modeling for realistic base capacitances exist, a comparison of the effective length ratios with those from the analytical inversion technique is worthwhile.

[42] So, parallel to the least squares method, an analytical inversion technique is developed. The main benefit of the analytical inversion is the fact that a single solution is obtained for each set of measurements. With the same restrictions to the induced voltage measurements on the RPWS antennas, the effective length vectors of the RPWS antennas are derived for each measured autocorrelation and cross-correlation set. Similar to the least squares analysis, we assume that parameters $Q$ and $U$ are zero; therefore a 
Table 5. Colatitude and Azimuth of the RPWS Antennas to be Used as Operational Values for the RPWS Direction-Finding Until Huygens Probe is Released

\begin{tabular}{lccc}
\hline & $u$ Antenna & $v$ Antenna & $w$ Antenna \\
\hline$h / h_{w}$ & 1.21 & 1.19 & 1.0 \\
$\theta$ & $108.3^{\circ}$ & $107.8^{\circ}$ & $29.3^{\circ}$ \\
$\phi$ & $17.0^{\circ}$ & $163.8^{\circ}$ & $90.6^{\circ}$ \\
\hline
\end{tabular}

residual linear polarization component may induce some bias on the results. The results of the analytical inversion technique are summarized in Table 4.

[43] As shown, all derived values for the colatitude and azimuth of each antenna gives a difference from $0.5^{\circ}$ up to $1.5^{\circ}$, which is in the range of the standard deviation in both methods. Moreover, we found that the effective length ratios as obtained from the two applied methods are practically the same: the $u$ and $v$ antenna are approximately 20 percent larger than the $w$ antenna and the $u$ antenna is only slightly larger than the $v$ antenna $\left(h_{u} / h_{w}=1.21\right.$ and $\left.h_{v} / h_{w}=1.19\right)$ within the inherent precision.

[44] Table 5 gives the final values of the effective length vectors to be used as operational values for the RPWS direction-finding until the Huygens probe is released. These values are averages from the results obtained with the two methods. Typical error bars are 0.5 to 1 degree on the angles and 0.02 to 0.05 on the length ratios.

[45] Finally, we note that the derived values for the effective length vectors will have to be revised after Huygens Probe release, right after the beginning of the Saturnian tour, since the Huygens Probe additionally influences the behavior of the antennas (see Table 1). Therefore after the probe is released to Titan it will be necessary to repeat the analysis. The same procedure as described here will be applied to observations of Saturnian radio emissions performed near apo-apsis. They will provide the corrections (expected to be small) to the "operational values of the antenna parameters to be used for the RPWS directionfinding",

\section{Appendix A: Aspects of Background Determination}

[46] In order to perform reliable DF analysis on RPWSHFR observations of Jupiter's radio emissions, the signal used should be only that coming from Jupiter. A frequencydependent background should thus be subtracted from the data, which includes galactic background noise and receiver noise $(\mathrm{Pg}+\mathrm{Pr})$. This background was derived as follows: (1) several intervals of a few hours were selected, all with a very low level of solar and planetary radio activity (i.e., far before and after the Jupiter flyby period) and a low level of spacecraft interference; (2) at each frequency, the statistical fluctuations of the background level appear as a well-defined gaussian distribution centered on the average value of $(\mathrm{Pg}+\mathrm{Pr})$ at that frequency.

[47] The background noise spectrum derived in this way is little dependent on the various HFR setups used (i.e., frequency range, temporal and spectral resolutions, etc.), which are taken into account by the "zero level" calibration step through which HFR data numbers are converted to
$\mathrm{V}^{2} / \mathrm{Hz}$ with a relative accuracy better than $1-2 \mathrm{~dB}$. Conversely, the dipole and monopole antennas (furthermore perturbed by the large spacecraft body) have very different spectral responses. Measurements performed with dipole and monopole antennas should then be processed separately. Figure A1 accordingly displays the resulting curves $(\operatorname{Pg}+\operatorname{Pr})(f)$ for measurements performed with $u$ and $v$ monopole antennas, which are those relevant for DF studies (similar curves are obtained for $u$ or $v$ separately).

[48] The main electrical resonance at $9.5 \mathrm{MHz}$ corresponds to the imaginary part of the antenna impedance becoming inductive and cancelling the base capacitance [see Zarka et al., this issue]. Secondary peaks also appear on the measured background spectrum. Some of them (at $3 \mathrm{MHz}$ and above $13 \mathrm{MHz}$ ) are due to quasi-permanent spacecraft interference, others (at 6.3 and $7.5 \mathrm{MHz}$ ) are secondary electrical resonances resulting from the interaction between the antenna and the conductive spacecraft structure, especially the $\approx 10 \mathrm{~m}$-long magnetometer boom placed symmetrically between the $u$ and $v$ monopoles. In the monopole mode the resonance frequency is found to vary with time (between 9.4 and $9.6 \mathrm{MHz}$ for the main one) and in amplitude (by a few $\mathrm{dB}$ ), probably due to moving parts on the spacecraft (as the Cosmic Dust Analyzer and soon the Huygens Probe). This constitutes a major limitation on the accuracy of the background determination. The internal HFR calibrations periodically carried out in-flight do not help in this case because the problem arises from the variation of the antenna response.

[49] In order to achieve better accuracy, it is thus necessary to compute the galactic spectrum measured at the time of the observations we want to calibrate. During the \pm 6 months around Jupiter's flyby, the nearly continuous

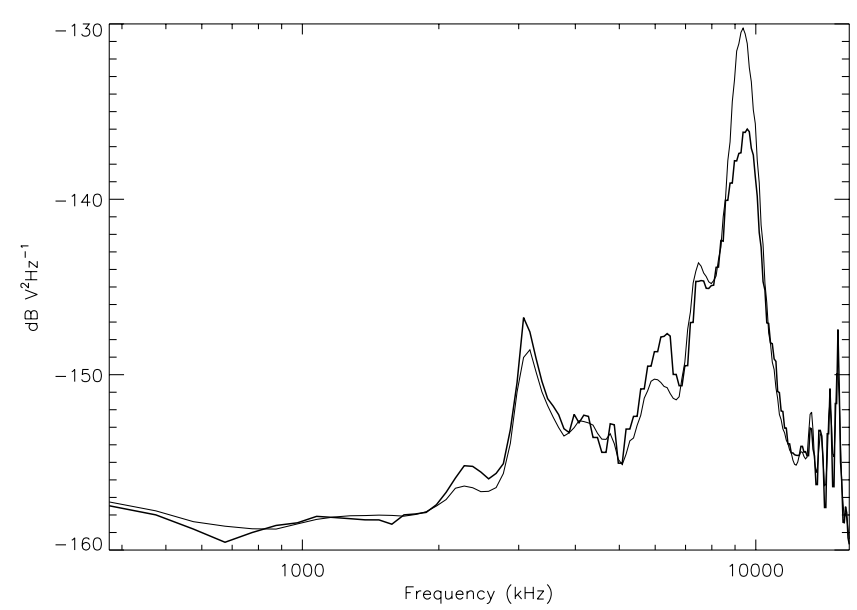

Figure A1. Background noise spectrum (including galactic plus receiver noise) measured by the RPWS-HFR with the monopole antennas during "quiet" intervals of a few hours far from Jupiter (lightface line). The boldface line was computed as the lower 5\% occurrence level detected at each frequency during a 6 month interval centered on the Jupiter flyby. It serves as our reference for the background spectrum $(\operatorname{Pg}+\operatorname{Pr})(f)$ for Jupiter studies. For more details, see Appendix A. 
Jovian radio activity does not leave room for quiet intervals of a few hours. However, we noticed that the limit of the lower $5 \%$ occurrence level in histograms of the distribution of intensities at each frequency, which takes advantages of quiet periods frequency per frequency and independent of their duration, is always close (within $1 \mathrm{~dB}$ ) of the galactic and receiver background level determined during the above "quiet" periods. Thus we compute in this way the background noise spectrum measured during the Jupiter flyby, which is displayed on Figure A1 as the boldface line. This plot confirms that significant variations are observed near the resonances. This latter spectrum (boldface) has been used as reference background $(\operatorname{Pg}+\operatorname{Pr})(f)$ over the Jupiter flyby period.

\section{Appendix B: Basic Equations for the Direction- Finding Analysis and Antenna Calibration}

[50] For deriving the wave information and the antenna parameters we first give the relevant equations. As discussed in section 3 , the real and imaginary parts of the model-predicted values are given as

$$
\begin{aligned}
\Re\left(\left\langle V_{i} V_{j}^{*}\right\rangle\right)= & \frac{S h_{i} h_{j}}{2}\left[(1+Q) \Omega_{i} \Omega_{j}-U \sin \theta_{i} \sin \left(\phi-\phi_{i}\right) \Omega_{j}\right. \\
& -U \sin \theta_{j} \sin \left(\phi-\phi_{j}\right) \Omega_{i}+(1-Q) \\
& \left.\cdot \sin \theta_{i} \sin \theta_{j} \sin \left(\phi-\phi_{i}\right) \sin \left(\phi-\phi_{j}\right)\right]
\end{aligned}
$$

and

$$
\Im\left(\left\langle V_{i} V_{j}^{*}\right\rangle\right)=\frac{S h_{i} h_{j}}{2} V\left[\sin \theta_{i} \sin \left(\phi-\phi_{i}\right) \Omega_{j}-\sin \theta_{j} \sin \left(\phi-\phi_{j}\right) \Omega_{i}\right],
$$

where $\Omega_{i}=\cos \theta_{i} \sin \theta-\sin \theta_{i} \cos \theta \cos \left(\phi-\phi_{i}\right)$. For the sake of simplicity we introduce $\Psi_{i}=\sin \theta_{i} \sin \left(\phi_{i}-\phi\right), S_{1}=S Q$, $S_{2}=S U$, and $S_{3}=S V$, respectively. Thus the equations (B1) and (B2) transform to

$$
\begin{gathered}
\left\langle V_{u} V_{u}^{*}\right\rangle=\frac{h_{u}^{2}}{2}\left[S\left(\Omega_{u}^{2}+\Psi_{u}^{2}\right)+S_{1}\left(\Omega_{u}^{2}-\Psi_{u}^{2}\right)+2 S_{2} \Omega_{u} \Psi_{u}\right], \\
\left\langle V_{v} V_{v}^{*}\right\rangle=\frac{h_{v}^{2}}{2}\left[S\left(\Omega_{v}^{2}+\Psi_{v}^{2}\right)+S_{1}\left(\Omega_{v}^{2}-\Psi_{v}^{2}\right)+2 S_{2} \Omega_{v} \Psi_{v}\right], \quad \\
\left\langle V_{w} V_{w}^{*}\right\rangle=\frac{h_{w}^{2}}{2}\left[S\left(\Omega_{w}^{2}+\Psi_{w}^{2}\right)+S_{1}\left(\Omega_{w}^{2}-\Psi_{w}^{2}\right)+2 S_{2} \Omega_{w} \Psi_{w}\right], \\
\Re\left(\left\langle V_{u} V_{w}^{*}\right\rangle\right)=\frac{h_{u} h_{w}}{2}\left[S\left(\Omega_{u} \Omega_{w}+\Psi_{u} \Psi_{w}\right)+S_{1}\left(\Omega_{u} \Omega_{w}-\Psi_{u} \Psi_{w}\right)\right. \\
\left.+S_{2}\left(\Psi_{u} \Omega_{w}+\Omega_{u} \Psi_{w}\right)\right],
\end{gathered}
$$

$$
\begin{aligned}
\Re\left(\left\langle V_{v} V_{w}^{*}\right\rangle\right)= & \frac{h_{v} h_{w}}{2}\left[S\left(\Omega_{v} \Omega_{w}+\Psi_{v} \Psi_{w}\right)+S_{1}\left(\Omega_{v} \Omega_{w}-\Psi_{v} \Psi_{w}\right)\right. \\
& \left.+S_{2}\left(\Psi_{v} \Omega_{w}+\Omega_{v} \Psi_{w}\right)\right] \\
& \Im\left(\left\langle V_{u} V_{w}^{*}\right\rangle\right)=\frac{h_{u} h_{w}}{2} S_{3}\left[\Omega_{u} \Psi_{w}-\Omega_{w} \Psi_{u}\right] \\
& \Im\left(\left\langle V_{v} V_{w}^{*}\right\rangle\right)=\frac{h_{v} h_{w}}{2} S_{3}\left[\Omega_{v} \Psi_{w}-\Omega_{w} \Psi_{v}\right]
\end{aligned}
$$

The retrieval of the parameters is based finding the weighted least squares sum of the difference between the wave observations, $y_{i}^{o b s}$, and the model-predicted values, $y_{i}^{\text {mod }}$, [Ladreiter et al., 1995]

$$
\chi^{2}=\sum_{i=1}^{N} \mathcal{W}_{i}\left[y_{i}^{o b s}-y_{i}^{\bmod }(\mathbf{X})\right]^{2}=\operatorname{Min}
$$

where the model-predicted values are described via equations (B3) through (B9). The weights $\mathcal{W}$ reflect the uncertainty of each modeled value, simply expressed via the corresponding standard deviations, $\mathcal{W}=1 / \sigma^{2}$. Note that $\sigma$ contains the intrinsic receiver noise level, $\Delta_{r e c}=10^{-16} \mathrm{~V}^{2} / \mathrm{Hz}$. For the direction-finding (DF) analysis, the retrieved vector $\mathbf{X}$ contains the wave parameters and for the antenna calibration (AC) $\mathbf{X}$ contains the effective length vectors.

\section{Appendix C: Direction-Finding Analysis Using the Least Squares Method}

[51] In the following we assume that the state of polarization does not change within the switching time of the receivers. Hence the autocorrelation $\bar{A}_{w w}$ is the mean value of autocorrelations of the two subsets, $\bar{A}_{w w}=\left(A_{w w}+\tilde{A}_{w w}\right) / 2$ (more explanations on $\bar{A}_{w w}$ are given in section 3 ). We further normalize the observations and the model-predicted values by the squared sum of autocorrelations. Hence equation (B10) reads

$$
\begin{aligned}
\chi^{2}= & \sum_{i}^{N}\left[\mathcal{W}_{1}\left(\frac{A_{u u}}{N^{o b s}}-\frac{\left\langle V_{u} V_{u}^{*}\right\rangle}{N^{\text {mod }}}\right)^{2}\right]_{i}+\left[\mathcal{W}_{2}\left(\frac{A_{v v}}{N^{o b s}}-\frac{\left\langle V_{v} V_{v}^{*}\right\rangle}{N^{\text {mod }}}\right)^{2}\right]_{i} \\
& +\left[\mathcal{W}_{3}\left(\frac{\bar{A}_{w w}}{N^{o b s}}-\frac{\left\langle V_{w} V_{w}^{*}\right\rangle}{N^{\text {mod }}}\right)^{2}\right]_{i}+\left[\mathcal{W}_{4}\left(\frac{C_{u w}}{N^{\text {obs }}}-\frac{\Re\left(\left\langle V_{u} V_{w}^{*}\right\rangle\right)}{N^{\text {mod }}}\right)^{2}\right]_{i} \\
& +\left[\mathcal{W}_{5}\left(\frac{C_{v w}}{N^{o b s}}-\frac{\Re\left(\left\langle V_{v} V_{w}^{*}\right\rangle\right)}{N^{\text {mod }}}\right)^{2}\right]_{i} \\
& +\left[\mathcal{W}_{6}\left(\frac{I_{u w}}{N^{o b s}}-\frac{\Im\left(\left\langle V_{u} V_{w}^{*}\right\rangle\right)}{N^{\text {mod }}}\right)^{2}\right]_{i} \\
& +\left[\mathcal{W}_{7}\left(\frac{I_{v w}}{N^{o b s}}-\frac{\Im\left(\left\langle V_{v} V_{w}^{*}\right\rangle\right)}{N^{\text {mod }}}\right)^{2}\right]_{i}
\end{aligned}
$$


where index $i$ counts the observations ( $N$ being the total number of observed autocorrelation and cross-correlations). The normalization factors are given by

$$
\begin{aligned}
N^{o b s} & =\sqrt{A_{u u}^{2}+A_{v v}^{2}+\bar{A}_{w w}^{2}}, \\
N^{m o d} & =\sqrt{\left\langle V_{u} V_{u}^{*}\right\rangle^{2}+\left\langle V_{v} V_{v}^{*}\right\rangle^{2}+\left\langle V_{w} V_{w}^{*}\right\rangle^{2}},
\end{aligned}
$$

and the weights are simply expressed as

$$
\begin{aligned}
& W_{1}=\left[\frac{\Delta_{r e c} \sqrt{\left\langle V_{v} V_{v}^{*}\right\rangle^{2}+\left\langle V_{w} V_{w}^{*}\right\rangle^{2}}}{\left\langle V_{u} V_{u}^{*}\right\rangle^{2}+\left\langle V_{v} V_{v}^{*}\right\rangle^{2}+\left\langle V_{w} V_{w}^{*}\right\rangle^{2}}\right]^{-2}, \\
& W_{2}=\left[\frac{\Delta_{r e c} \sqrt{\left\langle V_{u} V_{u}^{*}\right\rangle^{2}+\left\langle V_{w} V_{w}^{*}\right\rangle^{2}}}{\left\langle V_{u} V_{u}^{*}\right\rangle^{2}+\left\langle V_{v} V_{v}^{*}\right\rangle^{2}+\left\langle V_{w} V_{w}^{*}\right\rangle^{2}}\right]^{-2}, \\
& W_{3}=\left[\frac{\Delta_{r e c} \sqrt{\left\langle V_{u} V_{u}^{*}\right\rangle^{2}+\left\langle V_{v} V_{v}^{*}\right\rangle^{2}}}{\left\langle V_{u} V_{u}^{*}\right\rangle^{2}+\left\langle V_{v} V_{v}^{*}\right\rangle^{2}+\left\langle V_{w} V_{w}^{*}\right\rangle^{2}}\right]^{-2}, \\
& W_{4}=\left[\frac{\Delta_{\text {rec }} \sqrt{\left\langle V_{u} V_{u}^{*}\right\rangle^{2}+\left\langle V_{v} V_{v}^{*}\right\rangle^{2}+\left\langle V_{w} V_{w}^{*}\right\rangle^{2}+\Re\left(\left\langle V_{u} V_{w}^{*}\right\rangle\right)^{2}}}{\left\langle V_{u} V_{u}^{*}\right\rangle^{2}+\left\langle V_{v} V_{v}^{*}\right\rangle^{2}+\left\langle V_{w} V_{w}^{*}\right\rangle^{2}}\right]^{-2}, \\
& W_{5}=\left[\frac{\Delta_{r e c} \sqrt{\left\langle V_{u} V_{u}^{*}\right\rangle^{2}+\left\langle V_{v} V_{v}^{*}\right\rangle^{2}+\left\langle V_{w} V_{w}^{*}\right\rangle^{2}+\Re\left(\left\langle V_{v} V_{w}^{*}\right\rangle\right)^{2}}}{\left\langle V_{u} V_{u}^{*}\right\rangle^{2}+\left\langle V_{v} V_{v}^{*}\right\rangle^{2}+\left\langle V_{w} V_{w}^{*}\right\rangle^{2}}\right]^{-2}, \\
& W_{6}=\left[\frac{\Delta_{r e c} \sqrt{\left\langle V_{u} V_{u}^{*}\right\rangle^{2}+\left\langle V_{v} V_{v}^{*}\right\rangle^{2}+\left\langle V_{w} V_{w}^{*}\right\rangle^{2}+\Im\left(\left\langle V_{u} V_{w}^{*}\right\rangle\right)^{2}}}{\left\langle V_{u} V_{u}^{*}\right\rangle^{2}+\left\langle V_{v} V_{v}^{*}\right\rangle^{2}+\left\langle V_{w} V_{w}^{*}\right\rangle^{2}}\right]^{-2}, \\
& W_{7}=\left[\frac{\Delta_{r e c} \sqrt{\left\langle V_{u} V_{u}^{*}\right\rangle^{2}+\left\langle V_{v} V_{v}^{*}\right\rangle^{2}+\left\langle V_{w} V_{w}^{*}\right\rangle^{2}+\Im\left(\left\langle V_{v} V_{w}^{*}\right\rangle\right)^{2}}}{\left\langle V_{u} V_{u}^{*}\right\rangle^{2}+\left\langle V_{v} V_{v}^{*}\right\rangle^{2}+\left\langle V_{w} V_{w}^{*}\right\rangle^{2}}\right]^{-2} .
\end{aligned}
$$

Quantity $\chi^{2}$ (equation $(\mathrm{C} 1)$ ) is then iteratively minimized for the parameter vector $\mathbf{X}$ which contains the Stokes parameters $Q, U$, and $V$, and the directions of incidence of the received wave by using Powell's method [see Press et al., 1986]. The normalization is essential because it eliminates the Stokes parameter $S$, which can not be retrieved due to the unknown absolute magnitude of the effective antenna lengths (only the ratios $h_{u} / h_{w}$ and $h_{v} / h_{w}$ can be determined accurately).

\section{Appendix D: Antenna Calibration Mode Using the Least Squares Method}

[52] In the AC mode we consider the two subsets of measurements separately (allowing thus a change of the wave parameters between the two measurement times) and do not take into account equations (B8) and (B9) as they introduces the unknown parameter $V$. The first subset contains the RPWS measurements $A_{u u}, A_{w w}, C_{u w}$, and the second subset analogously $A_{v v}, \tilde{A}_{w w}, C_{v w}$. We assume no linear polarization, $Q=0$ and $U=0$ (justified by considerations of Ortega-Molina and Lecacheux [1991]), and the modeled autocorrelation and cross-correlations simply read

$$
\begin{gathered}
\left\langle V_{u} V_{u}^{*}\right\rangle=\frac{h_{u}^{2}}{2}\left[S\left(\Omega_{u}^{2}+\Psi_{u}^{2}\right)\right], \\
\left\langle V_{v} V_{v}^{*}\right\rangle=\frac{h_{v}^{2}}{2}\left[S\left(\Omega_{v}^{2}+\Psi_{v}^{2}\right)\right], \\
\left\langle V_{w} V_{w}^{*}\right\rangle=\frac{h_{w}^{2}}{2}\left[S\left(\Omega_{w}^{2}+\Psi_{w}^{2}\right)\right], \\
\Re\left(\left\langle V_{u} V_{w}^{*}\right\rangle\right)=\frac{h_{u} h_{w}}{2}\left[S\left(\Omega_{u} \Omega_{w}+\Psi_{u} \Psi_{w}\right)\right], \\
\Re\left(\left\langle V_{v} V_{w}^{*}\right\rangle\right)=\frac{h_{v} h_{w}}{2}\left[S\left(\Omega_{v} \Omega_{w}+\Psi_{v} \Psi_{w}\right)\right] .
\end{gathered}
$$

Hence the least squares sum is then given as

$$
\begin{aligned}
\chi^{2}= & \sum_{i}^{N}\left[\mathcal{W}_{11}\left(\frac{A_{u u}}{\sqrt{A_{u u}^{2}+A_{w w}^{2}}}-\frac{\left\langle V_{u} V_{u}^{*}\right\rangle}{\sqrt{\left\langle V_{u} V_{u}^{*}\right\rangle^{2}+\left\langle V_{w} V_{w}^{*}\right\rangle^{2}}}\right)^{2}\right]_{i} \\
+ & {\left[\mathcal{W}_{22}\left(\frac{A_{v v}}{\sqrt{A_{v v}^{2}+\tilde{A}_{w w}^{2}}}-\frac{\left\langle V_{v} V_{v}^{*}\right\rangle}{\sqrt{\left\langle V_{v} V_{v}^{*}\right\rangle^{2}+\left\langle V_{w} V_{w}^{*}\right\rangle^{2}}}\right]_{i}\right.} \\
+ & {\left[\mathcal{W}_{13}\left(\frac{C_{u w}}{\sqrt{A_{u u}^{2}+A_{w w}^{2}}}-\frac{\Re\left(\left\langle V_{u} V_{w}^{*}\right\rangle\right)}{\sqrt{\left\langle V_{u} V_{u}^{*}\right\rangle^{2}+\left\langle V_{w} V_{w}^{*}\right\rangle^{2}}}\right)^{2}\right]_{i} } \\
+ & {\left[\mathcal{W}_{23}\left(\frac{C_{v w}}{\sqrt{A_{v v}^{2}+\tilde{A}_{w w}^{2}}}-\frac{\Re\left(\left\langle V_{v} V_{w}^{*}\right\rangle\right)}{\sqrt{\left\langle V_{v} V_{v}^{*}\right\rangle^{2}+\left\langle V_{w} V_{w}^{*}\right\rangle^{2}}}\right)^{2}\right]_{i},(\mathrm{D} 6) }
\end{aligned}
$$

where the sum is over $N$ measurements, equations (D1)(D5) have to be taken into account, and the weights $\mathcal{W}_{11}$, $\mathcal{W}_{22}, \mathcal{W}_{13}$, and $\mathcal{W}_{23}$ are given as

$$
\mathcal{W}_{11}=\left[\frac{\Delta_{\text {rec }} \mid\left\langle V_{w} V_{w}^{*}\right\rangle}{\left\langle V_{u} V_{u}^{*}\right\rangle^{2}+\left\langle V_{w} V_{w}^{*}\right\rangle^{2}}\right]^{-2},
$$




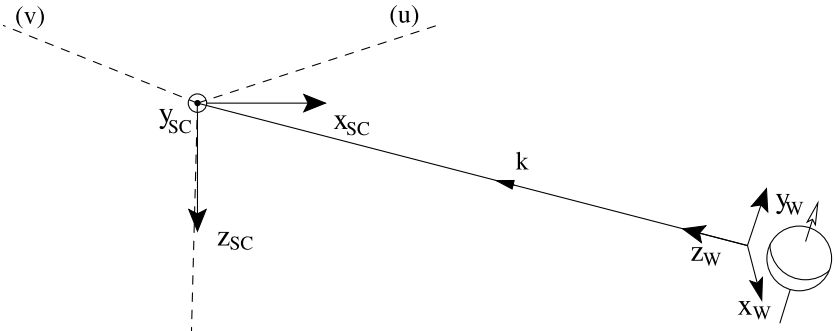

(w)

Figure E1. Wave frame $\left(\mathbf{x}_{w}, \mathbf{y}_{w}, \mathbf{z}_{w}\right)$ and spacecraft frame $\left(\mathbf{x}_{S C}, \mathbf{y}_{S C}, \mathbf{z}_{S C}\right)$. The antenna directions are shown in dashed lines. Quantity $\mathbf{z}_{w}$ is colinear to the wave vector $\hat{\mathbf{k}}$ and $\mathbf{y}_{w}$ is in the plane containing $\hat{\mathbf{k}}$ and the rotational axis of the observed planet.

$$
\mathcal{W}_{22}=\left[\frac{\Delta_{\text {rec }}\left|\left\langle V_{w} V_{w}^{*}\right\rangle\right|}{\left\langle V_{v} V_{v}^{*}\right\rangle^{2}+\left\langle V_{w} V_{w}^{*}\right\rangle^{2}}\right]^{-2},
$$

$$
\begin{aligned}
\mathcal{W}_{13}= & {\left[\Delta_{\text {rec }}\left|\frac{\Re\left(\left\langle V_{u} V_{w}^{*}\right\rangle\right)}{\sqrt{\left\langle V_{u} V_{u}^{*}\right\rangle^{2}+\left\langle V_{w} V_{w}^{*}\right\rangle^{2}}}\right|\right.} \\
& \left.\times \sqrt{\frac{1}{\left\langle V_{u} V_{u}^{*}\right\rangle^{2}+\left\langle V_{w} V_{w}^{*}\right\rangle^{2}}+\frac{1}{\Re\left(\left\langle V_{u} V_{w}^{*}\right\rangle\right)^{2}}}\right]^{-2}, \\
\mathcal{W}_{23}= & {\left[\Delta_{r e c}\left|\frac{\Re\left(\left\langle V_{v} V_{w}^{*}\right\rangle\right)}{\sqrt{\left\langle V_{v} V_{v}^{*}\right\rangle^{2}+\left\langle V_{w} V_{w}^{*}\right\rangle^{2}}}\right|\right.} \\
& \left.\times \sqrt{\frac{1}{\left\langle V_{v} V_{v}^{*}\right\rangle^{2}+\left\langle V_{w} V_{w}^{*}\right\rangle^{2}}+\frac{1}{\Re\left(\left\langle V_{v} V_{w}^{*}\right\rangle\right)^{2}}}\right]^{-2} .
\end{aligned}
$$

Again, $\chi^{2}$ from equation (D5) is minimized using Powell's method [see Press et al., 1986]. Here the retrieved parameter vector $\mathbf{X}$ contains the directions $\theta_{u}, \theta_{v}, \theta_{w}, \phi_{u}$, $\phi_{v}, \phi_{w}$ of the effective antenna axes and the antenna length ratios $h_{u} / h_{w}$ and $h_{v} / h_{w}$.

\section{Appendix E: Antenna Calibration Mode Using the Analytical Inversion Method}

[53] The analytical inversion method consists in solving analytically the equations (B3) to (B9) in order to derive wave parameters (if antenna parameters are known) or vice versa. For being able to do so, we have to find a suitable reference frame in which this set of equations can be simplified. As shown by Cecconi et al. (submitted manuscript, 2004), direction-finding (determination of wave parameters) can be done in the general case, i.e., whatever the wave polarization. By contrast, antenna calibration (determination of antenna parameters) can be done analytically only if one assumes the absence of wave linear polarization $(U=0, Q=0)$, and it consists in this case in solving a set of four equations (e.g., equations (B3), (B5), (B5), and (B8)). A critical step conditioning the accuracy of the results is an appropriate data selection (according to signal-to-noise ratio, polarization, and especially source position relative to the antennas), which depends on the specific antenna parameter to be determined. We present below the detailed equations leading to determination of antenna parameters. Direction-finding equations as well as an extensive study of the errors as a function of data selection are outside the scope of this paper. The interested reader is referred to Cecconi et al. (submitted manuscript, 2004).

[54] We start from equations (B3) to (B9). Assuming a purely circularly polarized wave $(U=0, Q=0), S_{1}$ and $S_{2}$ thus become zero and we obtain the following equations

$$
\left\langle V_{u} V_{u}^{*}\right\rangle=\frac{h_{u}^{2}}{2} S\left(\Omega_{u}^{2}+\Psi_{u}^{2}\right)
$$

$$
\left\langle V_{v} V_{v}^{*}\right\rangle=\frac{h_{v}^{2}}{2} S\left(\Omega_{v}^{2}+\Psi_{v}^{2}\right)
$$

$$
\begin{gathered}
\left\langle V_{w} V_{w}^{*}\right\rangle=\frac{h_{w}^{2}}{2} S\left(\Omega_{w}^{2}+\Psi_{w}^{2}\right), \\
\Re\left(\left\langle V_{u} V_{w}^{*}\right\rangle\right)=\frac{h_{u} h_{w}}{2} S\left(\Omega_{u} \Omega_{w}+\Psi_{u} \Psi_{w}\right), \\
\Re\left(\left\langle V_{v} V_{w}^{*}\right\rangle\right)=\frac{h_{v} h_{w}}{2} S\left(\Omega_{v} \Omega_{w}+\Psi_{v} \Psi_{w}\right), \\
\Im\left(\left\langle V_{u} V_{w}^{*}\right\rangle\right)=\frac{h_{u} h_{w}}{2} S V\left(\Omega_{u} \Psi_{w}-\Omega_{w} \Psi_{u}\right) \\
\Im\left(\left\langle V_{v} V_{w}^{*}\right\rangle\right)=\frac{h_{v} h_{w}}{2} S V\left(\Omega_{v} \Psi_{w}-\Omega_{w} \Psi_{v}\right) .
\end{gathered}
$$

[55] By working in the so-called wave frame (in which the $\mathrm{z}$ axis is the normalized wave vector, the $\mathbf{y}$ axis is in the plane containing the $\hat{\mathbf{k}}$ vector and Jupiter's rotation axis with orientation along the South-North direction and the $\mathbf{x}$ axis completes the right-hand triad; see Figure E1), we can write $\Omega_{i}=\sin \theta_{i} \cos \phi_{i}$ and $\Psi_{i}=\sin \theta_{i} \sin \phi_{i}$, where $\theta_{i}, \phi_{i}$, and $h_{i}(i=u, w)$ are the colatitude, azimuth, and length of the $i$ th antenna, respectively.

[56] We can simplify the above equations (E1) to (E7) so that an analytical inversion becomes possible. We discuss here the full solution of the set of four equations corresponding to the $(u, w)$ pair of antennas. Solution for the $(v, w)$ pair is obtained in the same way, replacing terms with index $u$ by the same terms with index $v$.

[57] In the wave frame we obtain thus the four following equations for the $(u, w)$ pair:

$$
\begin{aligned}
\left\langle V_{u} V_{u}^{*}\right\rangle & =S h_{u}^{2} \sin ^{2} \theta_{u}, \\
\left\langle V_{w} V_{w}^{*}\right\rangle & =S h_{w}^{2} \sin ^{2} \theta_{w},
\end{aligned}
$$




$$
\begin{aligned}
& \Re\left(\left\langle V_{u} V_{w}^{*}\right\rangle\right)=S h_{u} h_{w} \sin \theta_{u} \sin \theta_{w} \cos \left(\phi_{u}-\phi_{w}\right), \\
& \Im\left(\left\langle V_{u} V_{w}^{*}\right\rangle\right)=S V h_{u} h_{w} \sin \theta_{u} \sin \theta_{w} \sin \left(\phi_{u}-\phi_{w}\right),
\end{aligned}
$$

The ratio of the antennas' effective lengths is derived first, using as an input the rheometric values for antennas' azimuth and colatitude. The corresponding data selection must ensure here that the source direction is far from both antenna directions (i.e., $\alpha_{i}>30^{\circ}$; see definition of $\alpha_{i}$ in section 5.2). Smaller values of $\alpha_{i}$ increase the error on the result because the projection of the wave electric field on the antenna becomes small. We obtain thus

$$
h_{u} / h_{w}=\sqrt{\frac{A_{u u}}{A_{w w}} \frac{\sin ^{2} \theta_{w}}{\sin ^{2} \theta_{u}}} .
$$

[58] Derivation of antenna angles should then be done with different data selections for each antenna. For example, using the data selection appropriate for calibrating the $u$ antenna (as described in section 5.2) and assuming that we know the $w$ antenna parameters (for which rheometric accuracy is enough) and $h_{u} / h_{w}$, we solve the system for $S h_{w}^{2}, \theta_{u}, \phi_{u}$ and $V$ :

$$
\begin{gathered}
S h_{w}^{2}=\frac{A_{w w}}{\sin ^{2} \theta_{w}}, \\
\theta_{u}=\arcsin \left(\sqrt{\frac{A_{u u}}{A_{w w}} \frac{h_{w}^{2}}{h_{u}^{2}} \sin ^{2} \theta_{w}}\right), \\
\phi_{u}=\phi_{w}+\arccos \left(\frac{C_{u w}}{\sqrt{A_{w w} A_{v v}}}\right), \\
V=\frac{I_{u w}}{\sqrt{A_{w w} A_{u u}-\left(C_{u w}\right)^{2}}} \operatorname{sign}\left[\sin \left(\phi_{u}-\phi_{w}\right)\right] .
\end{gathered}
$$

Note that the absolute intensity flux $S$ cannot be derived but only its product with a reference antenna length squared, chosen to be $h_{w}^{2}$. From the same set of equations but using another data selection appropriate for the $w$ antenna calibration, we derive

$$
\begin{gathered}
S h_{w}^{2}=\frac{h_{w}^{2}}{h_{u}^{2}} \frac{A_{u u}}{\sin ^{2} \theta_{u}}, \\
\theta_{w}=\arcsin \left(\sqrt{\frac{A_{w w}}{A_{u u}} \frac{h_{u}^{2}}{h_{w}^{2}} \sin ^{2} \theta_{u}}\right), \\
\phi_{w}=\phi_{u}-\arccos \left(\frac{C_{u w}}{\sqrt{A_{w w} A_{u u}}}\right),
\end{gathered}
$$

$$
V=\frac{I_{u w}}{\sqrt{A_{w w} A_{u u}-\left(C_{u w}\right)^{2}}} \cdot \operatorname{sign}\left[\sin \left(\phi_{u}-\phi_{w}\right)\right] .
$$

In this case the angular parameters of the $u$ antenna as well as the ratio $h_{u} / h_{w}$ are assumed to be known. Note that analytical inversion provides one solution for each set of measurements and requires no iteration.

[59] Acknowledgments. Part of this work was done while D.F.V. and H.P.L. were on a research visit to the Observatoire de Paris, France, and D.F.V. was on a scientific visit to the University of Iowa, USA. We acknowledge support by the Austrian Academy of Sciences, by the Amadée project V2 from the "Österreichischer Austauschdienst" and by the University of Iowa. Cassini-RPWS activities at LESIA are supported by the French CNES (Centre National d'Etudes Spatiales). We owe much to Bob Manning, who passed away too soon (3 June 2002 at the age of 63).

[60] Arthur Richmond thanks Michael Kaiser and another reviewer for their assistance in evaluating this paper.

\section{References}

Born, M., and E. Wolf (1993), Principles of Optics, Pergamon, New York. Fischer, G., et al. (2001), Wire-grid modeling of Cassini spacecraft for the determination of effective length vectors of the RPWS antennas, in Planetary Radio Emissions V, edited by H. O. Rucker, M.-L. Kaiser, and Y. Leblanc, pp. 347-356. Austrian Acad. of Sci. Press, Vienna.

Gurnett, D. A., et al. (2004), The Cassini radio and plasma wave science investigation, Space Sci. Rev, in press.

Ladreiter, H. P., P. Zarka, and A. Lecacheux (1994), Direction finding study of Jovian Hectometric and broadband Kilometric radio emissions: Evidence for their auroral origin, Planet. Space Sci., 42, 919-931.

Ladreiter, H. P., P. Zarka, A. Lecacheux, W. Macher, H. O. Rucker, R. Manning, D. A. Gurnett, and W. S. Kurth (1995), Analysis of electromagnetic wave direction finding performed by spaceborne antennas using singular-value decomposition techniques, Radio Sci., 30, 1699-1712.

Ortega-Molina, A., and G. Daigne (1984), Polarization response of two crossed monopoles on a spacecraft, Astron. Astrophys., 130, 301-310.

Ortega-Molina, A., and A. Lecacheux (1991), Polarization of Jovian hectometric emission, J. Geophys. Res. 96, 11,441-11,453.

Press, W. H., B. P. Flannery, S. A. Teukolsky, and W. T. Vetterling (1986), Numerical Recipes, the Art of Scientific Computing, Cambridge Univ. Press, New York.

Rucker, H. O., W. Macher, R. Manning, and H. P. Ladreiter (1996), Cassini model rheometry, Radio Sci., 31, 1299-1311.

Zarka, P. (1998), Auroral radio emissions at the outer planets: Observations and theories, J. Geophys. Res., 103, 20,159-20,194.

Zarka P., B. Cecconi, and W. S. Kurth (2003), Jupiter's low frequency radio spectrum from Cassini/RPWS absolute flux density measurements, J. Geophys. Res., 109, A09S15, doi:10.1029/2003JA010260.

T. Averkamp, D. A. Gurnett, G. B. Hospodarsky, and W. S. Kurth Department of Physics and Astronomy, University of Iowa, Iowa City, IA, USA. (terrence-averkamp@uiowa.edu; donald-gurnett@uiowa.edu; george-hospodarsky@uiowa.edu; william-kurth@uiowa.edu)

B. Cecconi, P. Fédou, A. Lecacheux, and P. Zarka, Laboratoire d'Etudes Spatiales et d'Instrumentation en Astrophysique, Observatoire de Paris, Place Jules Janssen, 92195 Meudon Cedex, France. (baptiste.cecconi@ obspm.fr; pierre.fedou@obspm.fr; alain.lecacheux@obspm.fr; philippe. zarka@obspm.fr)

G. Fischer, H. P. Ladreiter, W. Macher, H. O. Rucker, and D. F. Vogl, Space Research Institute, Austrian Academy of Sciences, Schmiedlstrasse 6,8010 Graz, Austria. (georg.fischer@oeaw.ac.at; peter.ladreiter@ securitykag.at; wolfgang.macher@oeaw.ac.at; helmut.rucker@oeaw.ac.at; dieter.vog1@oeaw.ac.at) 